Sociologie et sociétés

SOCIOLOGIE

ET SOCIÉTÉS

\title{
Influence et solidarité, définir un fondement théorique à la
} sociologie

Influence and Solidarity, Defining a Conceptual Core for Sociology

\section{Victor LIDZ}

Volume 21, numéro 1, printemps 1989

Talcott Parsons : Relectures

URI : https://id.erudit.org/iderudit/001436ar

DOI : https://doi.org/10.7202/001436ar

Aller au sommaire du numéro

\section{Éditeur(s)}

Les Presses de l'Université de Montréal

\section{ISSN}

0038-030X (imprimé)

1492-1375 (numérique)

Découvrir la revue

Citer cet article

LIDZ, V. (1989). Influence et solidarité, définir un fondement théorique à la sociologie. Sociologie et sociétés, 21(1), 117-142.

https://doi.org/10.7202/001436ar

\section{Résumé de l'article}

Pendant les vingt dernières années de sa vie, Talcott Parsons a soutenu que l'objet d'étude essentiel de la sociologie devait être l'analyse de l'intégration sociale. S'inspirant pour une bonne part du modèle durkheimien de la solidarité, Parsons a conçu cette intégration sociale en termes dynamiques comme la capacité d'un groupe, d'une association ou d'une communauté à préserver sa cohésion lorsqu'ils se trouvent confrontés à des situations de désordre et de conflit. Les lacunes de la théorie de l'influence, théorie qu'il a placée au centre de son analyse de l'intégration sociale, expliquent en partie que Parsons ne soit pas parvenu à imposer ce programme de recherche à l'ensemble du milieu sociologique. Cet article s'efforce de reformuler la théorie de l'influence et de consolider ses fondements en envisageant les relations d'intégration comme étant de l'ordre de la rationalité. On propose un modèle comparable à celui de l'offre et de la demande. Enfin, on met en évidence les liens de ce modèle avec la théorie du groupe de référence et avec la théorie durkheimienne de la solidarité et des pathologies sociales. 


\section{Influence et solidarité, définir un fondement théorique à la sociologie}

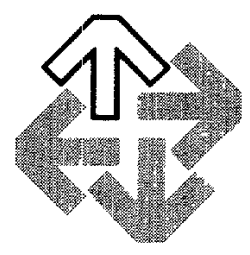

VICTOR LIDZ

\section{LE CADRE DE RÉFÉRENCE}

Talcott Parsons a élaboré son œuvre théorique sous le thème ambitieux de la théorie générale de l'action, ce qui dans son acception du terme impliquait deux objectifs audacieux. Le premier concerne l'étendue du champ d'application visée par la théorie. Parsons a conçu un cadre théorique qui soit susceptible de fournir des concepts d'analyse à chaque champ empirique dans le domaine de l'action sociale, aussi vaste soit-il. La théorie prétend être générale dans le sens où elle englobe tout ce qui constitue une conduite humaine significative. Le second but concerne l'étendue des orientations intellectuelles prises en compte par la théorie, à l'égard du monde de l'action sociale. Parsons a espéré pouvoir unifier dans un cadre théorique cohérent, solide sur le plan logique, les éléments reconnus pertinents de tous les schémas conceptuels établis avant lui pour l'étude de l'action sociale. La théorie devait être générale au sens où elle fournirait des outils théoriques fondamentaux à toute la communauté des chercheurs en sciences sociales, sans exclure aucune école ou aucun style de pensée jugé essentiel. Elle devait éviter à ces chercheurs le danger de construire une nouvelle tour de Babel où ils se trouveraient fatalement conduits, en s'engageant dans des théories logiquement inconciliables, à parler sans s'entendre et de là à tenter sans succès de résoudre leurs querelles ${ }^{1}$.

Parsons a cependant établi les limites qui circonscrivent ces deux ambitions par rapport à sa théorie générale de l'action. Par exemple, il a compris que certaines parties étaient restées dans une forme conceptuelle préliminaire. Ces parties se situent dans le cadre général de référence, mais on ne pouvait compter en établir complètement la valeur tant qu'elles ne seraient pas accompagnées de catégories analytiques plus précises et d'hypothèses d'explication plus spécifiques. Jusqu'à ce qu'un nombre important de champs empiriques aient été analysés en fonction de catégories plus précises et d'hypothèses

1. Sur la méthodologie employée par Parsons pour la théorie du développement, voir Victor Lidz, «Parsons and Empirical Sociology» dans Samuel Z. Klausner et Victor Lidz, The Nationalization of the Social Sciences, Philadelphia, University of Pennsylvania Press, 1986. Voir aussi Harold J. Bershady, Ideology and Social Knowledge, Oxford, Basil Blackwell, 1973. 
explicatives, la prétention à une validité générale resterait provisoire et purement formelle. Il ne s'agit pas de dire que pour Parsons l'importance du cadre général ait été négligeable, mais de remarquer qu'il en constatait simplement les limites. Parsons a également compris que sa démarche visant à déterminer les relations logiques de la théorie avec des corps théoriques indépendants, comme par exemple l'interactionnisme symbolique, l'ethnométhodologie, la psychanalyse et certaines variantes du marxisme, faisait intervenir des questions complexes ${ }^{2}$.

Conscient de ces difficultés, Parsons a travaillé au développement de sa théorie générale avec une certaine souplesse. L'élaboration de formulations techniques susceptibles d'apporter plus de précision analytique à des aspects spécifiques de la théorie lui a souvent donné l'occasion de revoir des concepts plus généraux. Il a également procédé à l'étude critique de théories concurrentes, ce qui lui a permis d'identifier des concepts intégrables à la théorie de l'action. En ayant l'ambition d'accéder à la généralité, il ne prétendait donc pas aboutir à un corpus théorique définitif, mais plutôt à un certain nombre de valeurs pouvant guider l'élaboration d'une meilleure théorie.

Tout au long de sa carrière, Parsons s'est penché en particulier sur la définition de la place de la théorie sociologique dans le champ multidisciplinaire plus vaste de la théorie de l'action. Dans le but précis de donner une orientation à la pensée sociologique, il a tenté d'établir des concepts fondamentaux et des hypothèses de base à l'intérieur d'une théorie plus générale. Pour résumer la manière dont il a abordé ce problème, on peut distinguer trois démarches qu'il a suivies à différents moments de sa carrière. La première est développée dans The Structure of Social Action. Il fixa à la sociologie la tâche de comprendre de façon globale l'intégration des systèmes de l'action sociale par le biais de valeurs communes ${ }^{3}$. Son souci premier était de démontrer que cette proposition codifiait les traditions de l'analyse comparative macro-institutionnelle établies par Weber et Durkheim. La seconde démarche, présentée dans The Social System, est étroitement reliée à la première, mais s'exprime en termes un peu plus généraux. La sociologie doit être centrée sur l'analyse du mode d'institutionnalisation des ordres normatifs ${ }^{4}$. Parsons a soutenu que cette hypothèse devrait faire le lien entre l'étude dynamique de l'interaction et des relations personnelles et l'analyse macrosociologique et institutionnelle, dont il avait souligné l'importance dans ses premiers travaux.

La troisième démarche est apparue à la fin des années cinquante et au début de la décennie suivante avec le paradigme à quatre fonctions et la notion de sous-systèmes sociaux différenciés par leur fonction. Dans ce schéma, les systèmes sociaux sont conçus comme étant organisés autour de quatre dimensions, distinctes sur le plan conceptuel mais reliées les unes aux autres: l'adaptation, la poursuite des buts, l'intégration et le maintien des modèles ${ }^{5}$. La sociologie est définie comme l'étude du sous-système de l'intégration des systèmes sociaux, tout en comprenant également l'étude des rapports de dépendance mutuelle de ce sous-système avec les trois autres. La recherche sociologique devrait se concentrer sur l'ensemble des structures et des processus de la vie sociale qui déterminent (ou sont déterminés par) la solidarité ou la cohérence sociale de toute relation empirique d'interaction. Du point de vue de la recherche macrosociologique, Parsons a analysé les institutions juridiques, les divers mécanismes du contrôle social et les relations entre les classes sociales et entre les groupes sociaux comme étant des facteurs majeurs

2. La compréhension de Parsons de ces problèmes a été fondée tout au long de sa carrière sur la méthode de la convergence développée dans son premier livre, The Structure of Social Action, New York, McGraw-Hill, 1937 et New York, Free Press, 1964 et 1968.

3. Ibid., chapitre XIX.

4. Talcott Parsons, The Social System, New York, Free Press, 1951, chapitre XII.

5. Voir Talcott Parsons, «An Outline of the Social System» dans T. Parsons, E. A. Shils, K. D. Naegele et J. R. Pitts, Theories of Society, New York, Free Press, 1961, pour une introduction au paradigme des quatre fonctions. 
qui déterminent le processus d'intégration. Ces facteurs étaient censés constituer le soussystème de l'intégration sociale, lui-même composé d'un ensemble de relations dont la fonction spécifique est de faire naître la solidarité dans la société tout entière ${ }^{6}$. C'est cette troisième démarche, dans la mesure où elle s'efforce de concevoir un schéma théorique central pour la sociologie, qui est le point de départ de notre travail: la notion de soussystème d'intégration sociale jouera donc un rôle important dans notre propos.

Pendant les vingt dernières années de sa vie, Parsons a approfondi cette troisième conception des tâches fondamentales de la sociologie. Durant cette période, il a écrit, avec toute la finesse qui le caractérise, sur bien des aspects de l'intégration sociétale, notarnment sur divers processus à l'œuvre dans le contrôle social, les institutions juridiques, la stratification sociale, les droits civiques et la solidarité dans la communauté sociétale ${ }^{7}$. Il a également développé une conception dynamique des processus d'intégration autour de la notion d'influence définie comme un médium généralisé de persuasion ${ }^{8}$. Parsons n'est toutefois pas parvenu, malgré ses travaux d'envergure, à faire de la question de l'intégration sociétale le fondement, unifié sur le plan conceptuel, du projet de la recherche sociologique. En dehors de sa vision «harmoniste» de la société, pour l'essentiel erronée, et de son incapacité à analyser le conflit social, lacunes qui n'étaient pas sans importance pour l'ensemble des sociologues, Parsons s'est heurté à deux difficultés réelles.

Il était lui-même très conscient de la première de ces difficultés. Il n'avait pas réussi à donner assez d'unité à sa pensée et de puissance d'analyse à son étude des institutions et de leur dynamique d'intégration, pour parvenir à attirer l'attention du milieu scientifique qui aurait vu se constituer là un objectif de recherche impossible à ignorer. Les écrits sur la notion d'influence conçue comme un médium généralisé étaient, comme il l'a luimême reconnu, bien moins satisfaisants que ses essais sur le pouvoir politique ${ }^{9}$. Ils ne donnaient pas la même impression d'une unité dynamique des processus empiriques qu'ils étaient censés décrire, pas plus qu'ils ne frappaient les lecteurs par des perspectives aussi nouvelles et éclairantes. Comme de nombreux critiques l'ont écrit, ces textes n'avaient pas la précision conceptuelle de son célèbre essai On the Concept of Political Power ou de ses analyses de la monnaie conçue comme le prototype du médium généralisé. Parsons savait également qu'il n'avait pas expliqué les articulations entre les principaux ensembles

6. Voir, par exemple, l'accent mis sur les catégories intégratives dans la présentation de la théorie de l'action dans Talcott Parsons, Societies, Evolutionary and Comparative Perspectives, Englewood Cliffs, New Jersey, Prentice-Hall, 1966 et dans The System of Modern Societies, Englewood Cliffs, New Jersey, Prentice-Hall, 1971. Parsons a mis davantage l'accent sur l'intégration dans le manuscrit auquel il travaillait au moment de sa mort, The American Societal Community (sous presse).

7. Voir Talcott Parsons, «A Revised Analytical Approach to the Theory of Social Stratification» dans ses Essays in Sociological Theory, édition revisée, New York, Free Press, 1954; «Social Strains in America», «Some Reflections on the Institutional Framework of Economic Development» et «The Principal Structures of Community» dans Structure and Process in Modern Societies, New York, Free Press, 1960; «Durkheim's Contribution to the theory of Integration of Social Systems» et «Some Comments on the Sociology of Karl Marx» dans Sociological Theory and Modern Society, New York, Free Press, 1967, et «Voting and the Equilibrium of the American Political System», «The Mass Media and the Structure of American Society» (avec Winston White) et «Full Citizenship for the Negro American?» dans Politics and Social Structure, New York, Free Press, 1969.

8. Voir «On the Concept of Influence» dans Politics and Social Structure, op. cit.

9. En conséquence, son premier essai sur l'influence, «On the Concept of Influence», a été suivi par des discussions approfondies sur les difficultés qu'il soulève, avec des étudiants gradués, des assistants de recherche et des collaborateurs. J'ai eu le privilège de participer à nombre de ces débats. Parsons a reformulé plusieurs aspects de sa théorie de l'influence durant les années qui suivirent. Voir «Postscript to Chapter 15», en annexe du premier essai sur l'influence lors de sa réédition dans Politics and Social Structure et «Polity and Society. Some General Considerations», le chapitre de conclusion de Politics and Social Structure et la discussion sur l'influence dans Talcott Parsons et Gerald M. Platt, The American University, Cambridge, Harvard University Press, 1973. L'analyse de l'influence dans The American Societal Community propose une nouvelle formulation, mais ne dissipe pas les doutes ressentis par Parsons lui-même sur nombre de problèmes. 
d'institutions intégratrices - le droit et les autres mécanismes du contrôle social, la stratification sociale et la communauté sociétale - en des termes pouvant exprimer la cohérence fonctionnelle d'un sous-système sociétal. L'idée que l'étude de l'intégration sociétale puisse constituer un champ d'investigation distinct et unifié n'a donc pas résisté à l'examen critique. The American Societal Community ${ }^{10}$, livre dont Parsons avait presque achevé le premier jet au moment de sa mort, était censé établir l'unité de ce champ de recherche. Sa pensée sur l'intégration sociétale, y compris les paradigmes à quatre fonctions qu'il a utilisés pour formaliser les relations entre ses propositions théoriques, n'ayant cessé d'évoluer au cours des sept années de la rédaction de ce manuscrit, on reste perplexe quant à ses visées de cohérence conceptuelle.

La seconde difficulté concerne l'ensemble de la théorie de l'action et son statut à l'intérieur de la sociologie. Parsons a perdu une grande partie de son audience précisément pendant la période où il espérait établir l'analyse de l'intégration sociale comme thème principal de la recherche sociologique. Il apparait rétrospectivement que l'évolution rapide de sa pensée dans la seconde moitié des années cinquante, et particulièrement la multiplication des niveaux d'analyse, des paradigmes à quatre fonctions et des hypothèses émises au sujet de divers médias généralisés, a dépassé la grande majorité des sociologues. Les premiers écrits avaient pu paraître d'un abord difficile, mais ils n'en donnaient pas moins aux lecteurs une idée claire de ce qu'était la visée de l'analyse sociologique. Par contre, aborder ses textes ultérieurs exigeait du lecteur la maîtrise d'un système théorique très technique avant de pouvoir saisir avec précision le cadre d'analyse que Parsons proposait de placer au centre de la sociologie. Avant qu'il ne soit question d'envisager une étude particulière, le simple fait de suivre le modèle des hypothèses spécifiques inscrites dans ce cadre de référence posait toute une série d'autres problèmes. La plupart des sociologues n'eurent pas la patience voulue et cherchèrent ailleurs une orientation théorique susceptible de les guider, en alléguant pour se justifier les critiques qu'on portait généralement à la pensée parsonienne: harmonisme, déviations structurelles, inattention portée au changement social et échec à rendre compte du conflit. Ces attaques apparaissaient tout à fait appropriées pour rejeter une théorie qui prétendait concentrer l'attention sur l'intégration sociétale.

Cet article porte sur les deux difficultés fondamentales que l'on vient d'exposer. Cependant, étant donné les limites qui nous sont imparties, nous nous bornerons à proposer une base de réflexion pour des recherches à venir. La question clé sera d'indiquer une voie à suivre pour reformuler la théorie de l'influence. Nous tenterons de relier la conception de l'influence, en tant que médium généralisé facilitant le processus d'intégration, avec une conception dynamique de la solidarité telle que Durkheim l'a pensée. Si l'on reprend les idées contenues dans les premiers écrits de Durkheim sur la solidarité, on parvient à une vision plus claire, plus précise, des centres d'intérêt essentiels de la théorie sociologique. Nous espérons ainsi pouvoir établir un paradigme central de recherche, au sens kuhnien ${ }^{11}$ du terme. Il n'est toutefois pas dans notre intention de réduire la théorie générale de l'action ou encore les visées intellectuelles de la sociologie à leur expression essentielle. Il s'agit plutôt de reconnaître la théorie dynamique de l'influence et de la solidarité comme point de départ de l'analyse. Cela devrait guider notre recherche, à partir des questions fondamentales sur les relations que la solidarité fait naître, vers une série d'autres problèmes concernant d'importantes institutions d'intégration: les articulations entre l'influence/solidarité et d'autres mécanismes dynamiques de la société (la monnaie et les marchés, le pouvoir et l'autorité, les engagements et les valeurs structurelles) et des questions psychosociales (c'est-à-dire, en termes plus techniques, le système général de l'action) concernant les

10. Le manuscrit de ce travail peut être examiné dans les papiers de Talcott Parsons conservés aux archives de l'Université d'Harvard. Je suis actuellement occupé à le réviser en vue d'une éventuelle publication.

11. Thomas S. Kuhn, The Structure of Scientific Revolutions, édition révisée, Chicago, University of Chicago Press, 1970. 
liens entre les relations de solidarité, en tant que réalités de l'expérience sociale, et des processus comme la culture, la personnalité et la pensée ${ }^{12}$. Le paradigme essentiel est censé fournir la souplesse nécessaire pour accéder à la fécondité analytique du schéma de l'action.

\section{LA THÉORIE DE L'INFLUENCE ET SES PROBLÈMES}

Sans en faire une critique générale, on doit signaler quelques aspects mis en lumière par l'analyse parsonienne de l'influence. Parsons rédigea son œuvre maîtresse sur la notion d'influence peu de temps après un article tout à fait novateur sur le pouvoir politique. Il a donc pu analyser la notion d'influence en se servant de la thèse majeure qu'il avait avancée dans son essai sur le pouvoir, à savoir que l'argent en tant que médium généralisé symbolique n'est pas un phénomène isolé, mais représente seulement un membre important d'une «famille» de média ${ }^{13}$. Dans son analyse critique sur les processus du politique, Parsons avait souligné l'importance du leadership comme source de flexibilité dans le fonctionnement des institutions politiques ${ }^{14}$. Cette analyse a toutefois mis également en évidence que le leadership n'est pas par essence une forme de pouvoir, pas plus d'ailleurs qu'un lieu de décision contraignante, mais plutôt un moyen plus souple d'inciter les citoyens à soutenir les initiatives du politique. Le leadership est une façon de chercher à mobiliser l'ensemble de la collectivité. Parsons l'a considéré comme une tentative pour exercer de l'influence sur le public (les membres de la société qui considèrent que ce qui touche le bien public relève de leurs rôles de citoyens et d'électeurs) et a suggéré que celui-ci soit considéré comme une institution de l'intégration sociétale plutôt que comme une institution de l'action directement politique ${ }^{15}$. Ses écrits sur le pouvoir et le politique ont ainsi conduit Parsons à dégager l'importance spécifique de l'influence considérée comme médium de l'intégration sociale.

L'hypothèse fondamentale de Parsons voulait que chacun des sous-systèmes de base de la société serve de «foyer» à un médium qui lui soit propre: l'économie à la monnaie, le politique au pouvoir, le système d'intégration (ou communauté sociétale) à l'influence, et le système de maintien des modèles (ou système fiduciaire) aux engagements moraux (value-commitments) $^{16}$. Cela n'empêche pas cependant que chaque médium circule à travers l'ensemble des quatre sous-systèmes. Parsons a étudié en détail les processus par lesquels les acteurs s'échangent quantités de médiums à partir des positions qu'ils occupent dans deux sous-systèmes différents, positions liées à leurs rôles sociaux réciproques et définies d'un point de vue institutionnel. Il a, par exemple, analysé les rapports du leadership et du soutien populaire comme un échange entre le politique et la communauté sociétale, échange qui révèle des parallèles intéressants avec la relation qui s'établit entre des entreprises commerciales dans le système économique et les ménages dans le système fiduciaire ${ }^{17}$. Le terme d'échange (interchange) a été utilisé pour souligner à la fois les

12. Sur ce dernier aspect de l'étude de la solidarité, on peut se reporter à un essai devenu classique de Rainer C. Baum, «The System of Solidarities» dans Indian Journal of Social Research, vol. XVI, $n^{\text {os }} 1$ et 2 , avril et août 1975, en hommage Talcott Parsons.

13. Voir «On the Concept of Influence», op. cit.

14. Talcott Parsons, «Voting and the Equilibrium», op. cit. et «On the Concept of Political Power» dans Politics and Social Structure, op. cit.

15. Ibid.

16. Une vue d'ensemble élémentaire de la théorie des médiums d'échange était présentée dans Talcott Parsons, «An Outline of the Social System», op. cit.

17. Talcott Parsons, «On the Concept of Political Power» et «On the Concept of Influence», op. cit. Pour l"analyse des relations entre les entreprises et les ménages, voir aussi Talcott Parsons et Neil J. Smelser, Economy and Society, New York, Free Press, 1956. Pour la théorie économique à l'arrière-plan de cette analyse, voir J. M. Keynes, The General Theory of Employment, Interest, and Money, London, MacMillan, 1936, et Frank Knight, The Economic Organization, New York, Kelley, 1951. 
implications systémiques de la circulation d'ensemble des médiums entre les sous-systèmes sociaux et les échanges spécifiques de médiums qui interviennent entre deux individus ou deux collectivités. Sur ce point, Parsons a élargi le modèle de l'analyse économique du courant circulaire (circular flow) entre une entreprise et un ménage, à un plus grand cercle d'échanges. Il a développé des hypothèses spécifiques à propos des conséquences que peuvent avoir certains déséquilibres dans la circulation d'ensemble des médiums en suggérant, par exemple, que si un leadership politique est incapable de recueillir un soutien populaire suffisant, cela peut mener à l'équivalent politique d'une dépression économique. Dans The American Societal Community, il a analysé les suites du «Watergate» comme une crise politique provoquée par un affaiblissement des relations d'influence entre les dirigeants et les électeurs.

Bien que dans le modèle parsonien, on insiste surtout sur la circulation des médiums d'échange, l'importance du «foyer» d'origine n'est pas négligée. De même que la monnaie permet de mesurer la valeur d'échange de toutes les marchandises, le pouvoir est conçu comme un moyen de déterminer la valeur courante des ressources politiques et l'influence, comme indicateur de la valeur d'intégration ${ }^{18}$. Il est évident que le pouvoir et l'influence ne peuvent être, comme la monnaie, quantifiés sur une échelle numérique. Toutefois, dans le sens commun, les membres de la société se font aisément une idée du degré plus ou moins grand de pouvoir ou d'influence que détiennent les différents acteurs sociaux. Les gens prennent quotidiennement des décisions sur les «quantités» de pouvoir ou d'influence qu'ils sont prêts à exercer dans des situations sociales données. Les gens ont également l'habitude d'évaluer dans quelle mesure les autres acteurs ont gagné ou perdu du pouvoir et de l'influence selon qu'ils ont réussi ou non à modifier leurs positions et leurs modes de participation dans des réseaux de relations sociales. À un niveau plus profond, le pouvoir et l'influence, comme mesures de valeur, servent à déterminer le type ou la qualité des ressources sociales appropriées au contexte des relations politiques ou au contexte des relations d'intégration. Par exemple, le principe civique qui veut qu'une personne riche qui a perturbé l'ordre public ne puisse acheter le silence de la presse représente la limite normative entre l'argent et l'influence. Les interdits, plus ou moins forts selon les sociétés ou les groupes sociaux, portant sur l'achat de charges publiques ou la corruption des fonctionnaires, touchent pour leur part la frontière entre la richesse et le pouvoir. Le fait qu'un ou une fonctionnaire ne puisse avoir un contrôle sur la façon dont il ou elle sera mis en question par la presse marque la limite entre le politique et le sous-système d'intégration. Ces limites reposent en partie sur une notion quantitative des médiums d'échange.

En développant sa conception de l'influence, Parsons a fait un effort particulier pour créer des liens avec les recherches théoriques et empiriques précédentes. Les études de la Columbia School sur les groupes de référence, l'influence personnelle et les changements d'opinions, effectuées par Merton, Lazarsfeld et leurs collègues, ont fourni à Parsons cet arrière-plan théorique ${ }^{19}$. Ayant tout d'abord réanalysé ces données dans Voting, un essai majeur sur le politique ${ }^{20}$, Parsons s'est ensuite consacré à l'examen attentif du champ de «la recherche sur l'opinion» que l'on trouve dans Personal Influence ${ }^{21}$. Il a suivi non

18. Voir «On the Concept of Political Power» et «On the Concept of Influence», op.cit.

19. Par exemple, Robert K. Merton, «Continuities in the Theory of Reference Groups and Social Structure» et «Patterns of Influence: Local and Cosmopolitan Influentials», chapitres IX et X dans Social Theory and Social Structure, éd. révisée, New York, Free Press, 1957; P. F. Lazarsfeld, S. R. Berelson et Hazel Gaudet, The People's Choice, seconde édition, New York, Columbia University Press, 1948; B. R. Berelson, P. F. Lazarsfeld et W. N. McPhee, Voting, Chicago, University of Chicago Press, 1954.

20. «Voting and the Equilibrium», op. cit.

21. Elihu Katz et Paul Lazarsfeld, Personal Influence, New York, Free Press, 1955. Curieusement, ce travail n'est pas cité dans l'essai de Parsons sur l'influence. Mais dans son enseignement et dans ses discussions personnelles à propos de l'influence, Parsons s'y référait explicitement comme une source de première importance dans ses travaux théoriques de la fin des années 1950 et du début des années 1960. 
seulement la voie de Lazarsfeld et de certains autres chercheurs de Columbia, mais aussi celle de son collègue d'Harvard, Samuel A. Stouffer ${ }^{22}$, lorsqu'il a employé le concept d'«opinion» pour désigner la catégorie des variables dépendantes de la théorie de l'influence. En d'autres termes, il a conçu ces objets d'étude comme des opinions qui disposent les individus à agir dans un sens particulier. Pour expliquer les changements d'opinion, il a pu recourir à la théorie du groupe de référence, à des concepts tels que celui des pressions contradictoires (cross-pressures) et à des hypothèses comme celle des «deux échelons du courant d'influence» qui établissent, par l'intermédiaire de «groupes primaires», une relation entre les dirigeants publics ou les sources d'information et les individus ${ }^{23}$.

Parsons a ajouté à cet appareil conceptuel l'idée que l'influence circule dans la société sous la forme d'un médium généralisé. Il a cherché à démontrer que l'influence pouvait être considérée comme une capacité généralisée à persuader ou à modifier les opinions ${ }^{24}$. Un acteur qui détient de l'influence dispose d'une ressource qu'il peut utiliser pour faire avancer des intérêts spécifiques en amenant les autres à accepter ses opinions. De la même manière, un acteur influent qui encourage les autres à changer d'opinion intervient le plus souvent sur les motivations de leur conduite future. Les personnes influentes en général ont l'avantage de pouvoir obtenir la coopération des autres et, le cas échéant, de les empêcher de coopérer avec une tierce partie. Compte tenu de ces avantages pratiques que procure l'influence, les sociologues devraient pouvoir examiner sa répartition totale entre les individus, les groupes, les associations et les communautés à l'intérieur de la société. Les sociologues devraient également pouvoir se pencher sur les relations à travers lesquelles l'influence s'exerce et sur les processus qui peuvent modifier sa répartition. Les contraintes systémiques et macrosociales qui pèsent sur la façon dont l'influence est attribuée et employée sont apparues plus clairement à partir de la perspective parsonienne. On a pu, par exemple, rattacher les modes de répartition de l'influence à la structure des groupes de statut et en relier les usages aux mécanismes du contrôle social. C'est l'éclairage que Parsons a donné à la notion d'influence qui est à l'origine de la tendance à intégrer l'étude de l'influence à une recherche plus générale sur la stratification sociale.

Remarquons rapidement les quelques difficultés rencontrées par Parsons dans sa tentative d'analyser l'influence comme un médium généralisé de l'intégration sociale. Tout d'abord le concept d'opinion est assez flou et tend à revêtir une signification psychologique ou sociopsychologique qui s'écarte d'une approche théorique axée sur les relations strictement sociales. À cet égard, le concept d'opinion suscite le même type de difficultés que celui d'attitude dans la tradition des travaux de G. H. Mead. Il s'agit là essentiellement d'un problème de niveau d'analyse, car il y a peu de doute sur le fait que les opinions et les attitudes existent et que leurs changements exigent des explications d'ordre sociopsychologique. Toutefois, comme l'on pourra plus facilement le constater quand Parsons, un peu plus tard, introduira une distinction entre les médiums du système social et les médiums du système général de l'action ${ }^{25}$, les changements d'opinion ou

22. Le travail qui l'a le plus immédiatement influencé était de Samuel A. Stouffer, Communism, Conformity, and Civil Liberties, New York, Doubleday, 1955, mais The American Soldier, 3 vol., par Samuel A. Stouffer et al., Princeton, New Jersey, Princeton University Press, 1949, a été, à l'origine, une importante source de recherche sur l'influence et le changement d'opinion.

23. Elihu Katz et Paul Lazarsfeld, Personal Influence, op. cit. Pour Parsons, une autre source clé sur l'importance des groupes primaires dans l'intégration sociale a été l'article d'Edward A. Shils, «The Study of the Primary Group» dans Daniel Lerner et Harold D. Lasswell, dir., The Policy Sciences, Stanford, Californie, Stanford University Press, 1951

24. Talcott Parsons, «On the Concept of Influence», op. cit.

25. Voir Talcott Parsons, «Some Problems of General Theory in Sociology», dans Social Systems and the Evolution of Action Theory, New York, Free Press, 1977, et aussi le chapitre 2 intitule «The Cognitive Complex: Knowledge, Rationality, Learning, Competence, Intelligence» dans T. Parsons et G. M. Platt, The American University, op. cit.; également Victor Lidz, «Introduction to General Action Analysis» dans J.J. 
d'attitude ne sont pas les processus empiriques sur lesquels l'intégration sociétale s'appuie le plus directement. Celle-ci repose plutôt sur les changements dans les relations sociales de solidarité, changements qui, dans le cadre de la théorie de l'influence, doivent être les premiers objets de recherche soumis à l'explication.

Deuxièmement, une difficulté du même ordre s'élève avec le concept de «persuasion». Trop de choses peuvent avoir un effet de persuasion dans la vie sociale. Le terme connote donc un processus flou, faisant entrer en jeu toute une série de facteurs. La coercition peut faire changer d'opinions, mais aussi différents processus culturels, y compris les démonstrations scientifiques, les fleurs de rhétorique et l'expression artistique. Si l'on étend la recherche à la totalité de ce champ de la persuasion, on risque de s'éloigner de la question centrale qui porte sur les relations et les processus de l'intégration.

Une troisième difficulté est apparue quand Parsons a relié la théorie de l'influence à ses propres études sur la profession médicale et, plus tard, sur les carrières académiques ${ }^{26}$. L'analyse des structures professionnelles a mis nettement en évidence le rôle généralisé de l'influence. Cette généralisation peut se manifester dans des situations dont on peut multiplier les exemples: un patient acceptant les conseils d'un médecin sur un traitement à suivre sans avoir la connaissance suffisante pour juger de leur valeur; un étudiant qui reçoit les enseignements d'un professeur sans pouvoir évaluer leur qualité. Dans les deux cas, l'influence est liée au statut professionnel (et aux diplômes) de celui qui en use. L'exercice de l'influence établit des liens là où existe ce que Parsons a nommé une différence de niveau de compétence ou d'information. Selon Parsons, dans le domaine professionnel, l'influence se fonde sur la compétence et réside dans la capacité généralisée à «persuader» à partir de l'écart des connaissances ${ }^{27}$. Quoique très éclairante, cette formulation ne tient pas compte d'un certain nombre de facteurs importants. Le rôle de médecin et celui de professeur, par exemple, entraînent, en plus de la maîtrise d'un savoir technique de haut niveau, une responsabilité envers le bien-être des patients ou des étudiants. D'autres types d'influence, celle des chefs de file politiques ou communautaires, par exemple, se fondent sur des responsabilités qui n'exigent pas forcément un savoir plus grand. Que l'influence publique du président Reagan ait été si peu affectée par l'étalage de son ignorance sur des questions sociales de première importance en constitue la preuve. Par conséquent, la notion de différence de niveau de compétence ne rend compte que partiellement du mode de généralisation de l'influence. Parsons a eu tendance à tirer des conclusions beaucoup trop larges à partir de traits spécifiques aux relations professionnelles.

En définitive, Parsons n'a pas réussi à construire le concept d'influence tout à fait sur le modèle de la monnaie. Comme il l'a lui-même souvent mentionné, les économistes ont mis l'accent sur le fait qu'une quantité d'argent donnée représente une part de la productivité de l'ensemble de l'économie. Selon ce modèle, l'influence ne devrait pas être uniquement reliée à la faculté de persuasion, mais à l'idée d'une capacité à créer des liens de solidarité dans une société. Les écrits de Parsons sur l'intégration sociétale montrent bien qu'il avait cette conception de l'influence. Loin de présumer, comme d'autres chercheurs l'ont souvent prétendu, que la société est harmonieuse à l'état naturel,

Lanbser, A. Effrat, R.C. Baum et V. Lidz (directeurs), Explorations in General Theory in Social Science, New York, Free Press, 1976 et Victor Lidz, «Transformational Theory and the Internal Environment of Action Systems» dans K. Knorr-Cetina et A. V. Cicourel, Advances in Social Theory and Methodology: Toward an Integration of Micro- and Macro-Sociologies, London, Routledge et Kegan Paul, 1981.

26. Talcott Parsons, «The Sick Role and the Role of the Physician Reconsidered», «Research with Human Subjects and the "Professional Complex"» et «Health and Disease: A Sociological and Action Perspective» dans Action Theory and the Human Condition, New York, Free Press, 1978; voir également T. Parsons et G. M. Platt, The American University, op. cit.

27. Voir aussi Talcott Parsons, «Professions» dans International Encyclopedia of the Social Sciences, New York, Free Press - Macmillan, 1968. 
Parsons savait que les sociétés sont porteuses de conflits et de tensions que seules des institutions actives d'intégration sociale peuvent corriger $^{28}$.

L'argumentation qui suit part de l'idée que l'influence désigne la capacité généralisée à prendre part aux processus de création et de formation de liens de solidarité, processus difficiles, susceptibles d'échouer à chaque étape de leur développement. On se référera aux thèses de Durkheim sur la cohésion des sociétés et leurs pathologies afin de pouvoir conceptualiser les conditions sociales qui influent sur le développement de la solidarité. Bien qu'elle doive être affinée, c'est néanmoins la notion parsonienne d'influence, considérée comme un «médium d'échange» de l'intégration sociale, qui constituera le fondement de notre analyse.

\section{L'ACTEUR RATIONNEL DANS LES STRUCTURES D'INTÉGRATION}

La théorie économique est fondée sur le modèle de l'acteur rationnel qui cherche à maximiser ses biens ou à contrôler le mieux possible les moyens de parvenir à ses buts. Comme Weber l'a montré il y a des années, ce modèle établit un idéal-type d'acteur ayant des motivations rationnelles ${ }^{29}$, permettant ainsi de comprendre en termes théoriques certains comportements couramment observés. Cela ne signifie pas que des personnes réelles vont nécessairement suivre ces comportements rationnels dans des circonstances particulières de leur vie. Parsons a également mis en garde, à plusieurs reprises, contre les dangers qui consisteraient à réifier, dans un cadre conceptuel immuable, les prémisses utilitaristes concernant l'acteur rationnel. Il a souligné que ces conceptions utilitaristes font obstacle à la compréhension des facteurs institutionnels qui déterminent la vie économique $^{30}$. Il a insisté sur le fait que toute tentative visant à fonder la sociologie sur des bases utilitaristes était vouée à l'échec; il s'est également opposé à la nouvelle économie politique des années 1960 et $1970^{31}$, ainsi qu'à la nouvelle sociologie économique de ces mêmes années $1970^{32}$. Ces derniers courants de pensée lui sont apparus comme une forme d'impérialisme intellectuel exercé par les économistes utilitaristes. Malgré leurs ambitions et leur intelligence, Parsons considérait qu'ils avaient une vision étroite des objectifs de la vie humaine. En dépit des réserves qu'il a émises sur la portée du modèle de l'acteur économique, «l'homme économique», Parsons a tout à fait reconnu que ce modèle était un point de départ utile pour la théorie économique en elle-même ${ }^{33}$ : il fournit

28. Ce point constitue la principale charge retenue par Parsons lors de sa critıque des thèses de Durkheim dans The Structure of Social Action, op. cit. et dans «Durkheim's Contribution to the Theory of Integration of Social Systems», op. cit. C'est aussi l'un des thèmes traités dans ses essais «Social Strains in America», «A Revised Analytical Approach to the Theory of Social Stratification» et «Full Citizenship for the Negro American?», op. cit.

29. Max Weber, Economy and Society, New York, Bedminster, 1968; première partie, chapitre II, «Socjological Categories of Economic Action».

30. Talcott Parsons, The Structure of Social Action, op. cit. et Parsons et Smelser, Economy and Society, op. cit.

31. Par exemple, Parsons a examiné et critiqué pour son propre compte des travaux comme ceux de Robert A. Dahl, A Preface to Democracy, Chicago, University of Chicago Press, 1957; Anthony Downs, An Economic Theory of Democracy, New York, Harper, 1957; James M. Buchanan et Gordon Tullock, The Calculus of Consent, Ann Arbor, Michigan, University of Michigan Press, 1965.

32. Par exemple, le livre de Gary Becker, The Economic Approach to Human Behavior, Chicago, University of Chicago Press, 1976, est un travail que Parsons a jugé erroné bien qu'il ait reconnu qu'il relevait d'un esprit intelligent et créatif.

33. Mark Gould (lors d'une conversation) a récemment soutenu que Parsons a fait une trop grande concession à l'économie néo-classique en admettant ce point. En fondant son argumentation sur des critiques récentes du cadre néo-classique au sein des sciences économiques elles-mêmes, aussi bien que sur sa propre critique du recours aux perspectives de Parsons et de Marx en sociologie, Gould suggère maintenant la nécessité d'un jugement plus radical des notions utilitaristes en économie. Il doit exposer ce point de vue dans une série d'essais à paraître. Je vais présenter ma théorie de la solidarité dans des termes qui sont en accord avec 
en effet une énonciation précise des prémisses de base permettant de systématiser un corps théorique complexe.

Dans le même esprit, nous allons exposer à grands traits les éléments d'un modèle de l'acteur de l'intégration ou de la solidarité. Ce modèle devrait être considéré, dans le meilleur des cas, comme un premier énoncé d'hypothèses sur la manière dont les acteurs sociaux, pour autant qu'ils agissent rationnellement, défendent au mieux leurs intérêts dans les relations d'intégration et dans les institutions ${ }^{34}$. Il n'est donc pas question de considérer que ce modèle puisse être à la base d'une explication totale de la conduite de solidarité ou des processus d'intégration. Il sera nécessaire, pour y parvenir, de compléter ce modèle par des analyses institutionnelles du type de celles que Parsons a identifiées pour établir les bases de l'économie néo-classique ${ }^{35}$. Nous ne devrions pas non plus présumer que les acteurs sociaux engagés dans des liens de solidarité agissent nécessairement sous l'empire de motivations rationnelles. Le modèle fournit néanmoins une base à partir de laquelle on pourra développer des hypothèses sur la dynamique ou le fonctionnement des mécanismes d'intégration dans la société, comme le modèle de l'acteur économique conduit à des hypothèses sur les marchés. Il établit aussi des points de référence pour tenter d'expliquer comment les relations et les mécanismes d'intégration se différencient de ceux de l'économie. En soulignant les différences par rapport à la théorie économique, on se démarque du même coup des théories utilitaristes en sociologie, dans la mesure où elles adoptent le même type de présupposés sur la rationalité de la conduite humaine.

$\mathrm{Si}$ les acteurs économiques cherchent à acquérir un maximum de biens, les acteurs de la solidarité recherchent le maximum d'avantages à tirer de leurs alliances avec les autres dans la société. Lorsqu'ils s'engagent dans des liens de solidarité, ces acteurs sont en mesure d'entraîner les autres dans une position d'obligation à leur égard. Quand ils ont besoin de soutien social, ils peuvent alors faire jouer ces obligations. On peut considérer l'influence comme un médium généralisé servant à engendrer et à maintenir les obligations qui sont attachées à des relations de solidarité de base. L'influence sert également à mesurer la «quantité» de pouvoir social nécessaire pour susciter des obligations, comme la monnaie est à la fois une mesure de la richesse et un moyen de se procurer des biens. Les acteurs chercheront donc à acquérir de l'influence à travers divers mécanismes relationnels, exactement comme ils sont portés à chercher de l'argent. Détenir de l'influence procure des avantages de tous ordres dans la gestion de la vie sociale. Une personne influente maîtrise la faculté d'inciter les autres acteurs à remplir leurs devoirs et leurs obligations. On pense souvent que de telles obligations sociales sont dues à des individus particuliers, mais l'influence est toujours détenue à l'intérieur de groupes et en général pour leur compte, pour celui d'associations ou de communautés. Il est donc important de penser que l'influence est ancrée dans les relations de solidarité et qu'elle constitue une mesure ou tout au moins un indicateur de la force de cette solidarité. On ne devrait pas considérer l'influence comme l'apanage d'individus uniquement.

l'évaluation parsonienne la plus ancienne concernant les prémisses de la théorie économique. Je réserve mon jugement sur ce que la critique de Gould des sciences économiques peut imposer comme changements dans la formulation de la théorie de la solidarité. Il pourrait s'avérer que de reconnaître la solidarité comme problématique en ce qui concerne les questions de l'utilité dans chaque relation économique concrète soit une autre façon de prendre partiellement en compte l'argument de Gould.

34. Mes formulations peuvent être comparées à la théorie utilitariste développée par Michael Hechter dans Principles of Group Solidarity, Berkeley et Los Angeles, University of California Press, 1987. La précision des thèses d'Hechter les rend séduisantes, mais elles restent sujettes à toutes les restrictions que Parsons a identifiées dans l'utilitarisme. Les critiques d'Hechter à propos des «normativistes», qui inclueraient les théoriciens de l'action, sont de l'ordre de la parodie simpliste. Elles ne tiennent pas vraiment compte des principaux arguments de The Structure of Sacial Action ou d'autres travaux parsoniens de première importance cités par Hechter. Hechter ne cite pas ou ne semble pas bien connaître les principaux écrits de Parsons sur l'influence, la solidarité ou les institutions d'intégration.

35. Voir Parsons et Smelser, Economy and Society, op. cit. 
De par leur nature même, les relations de solidarité sont réciproques. Elles lient entre eux les acteurs sociaux et, de là, elles situent chacun d'eux dans un ensemble organisé de devoirs envers l'autre ou envers les autres ${ }^{36}$. Les individus engagés dans ce type de relations de solidarité gagnent ainsi de l'influence les uns sur les autres. L'influence contient néanmoins des asymétries tout à fait caractéristiques, non seulement parce que certains acteurs en ont plus que d'autres, mais aussi parce que les niveaux d'influence sont étroitement liés aux différences dans leurs rôles sociaux ${ }^{37}$. Les individus particuliers n'auront de l'influence que pour faire intervenir un certain type d'obligations reconnues par les autres. L'influence, par exemple, qu'une personne peut exercer sur son conjoint médecin est différente de celle que pourraient exercer sur lui des patients ou des collègues et vice versa. Puisque les liens de solidarité sont réciproques, les acteurs sont amenés à comprendre la complexité et les différences qui existent dans le milieu qui est le leur et où peuvent s'exercer différentes influences. C'est son bon sens qui indique à un acteur qu'il subit des influences diverses. Il est probable, pour cette raison, que les acteurs sociaux seront conscients de la rareté de la ressource que représente l'influence et qu'ils seront sensibles aux types et aux degrés d'influence qu'ils n'ont pas. Leur rationalité les motivera à être déterminés à acquérir de l'influence et prudents dans la manière d'en user. Tous les membres de la société savent par expérience que l'on peut laisser passer une occasion d'acquérir de l'influence et par la suite être en situation de le regretter, tout comme l'on peut, par ailleurs, en gaspiller inutilement ou sans but précis. Dans toutes les cultures, il existe des maximes relatives à la manière d'acquérir de l'influence et de s'en servir rationnellement.

L'acteur qui utilise son influence et tire ainsi profit des obligations remplies par les autres, obtient un type particulier de bien, différent du bien économique. L'acteur bénéficie du soutien social indépendamment des à-côtés pratiques - acquisition de pouvoirs ou gains économiques. Ce soutien est la reconnaissance, ou mieux, la confirmation de la position de l'acteur dans un réseau de solidarité et constitue la réponse des autres à sa capacité d'activer les processus de coordination collective d'une conduite sociale. C'est, à un certain degré, l'affirmation du jugement personnel. C'est l'acceptation de la participation de l'acteur à un ensemble, un tout solidaire. C'est une expérience de la participation et de la communauté ${ }^{38}$. Étant donné que les membres ont des exigences les uns envers les autres dans le déroulement de la vie d'une entité de solidarité, les réactions suscitées par les différentes manières d'utiliser l'influence révèlent la capacité d'évolution de la collectivité en tant que réalité sui generis.

La confiance ou la méfiance, l'honnêteté ou la manipulation, la bonté ou la cruauté, l'optimisme débordant ou le pessimisme réducteur, entre autres, apparaissent comme autant de traits caractéristiques des relations collectives. On devrait donc considérer que tout ce que l'influence apporte aux individus fait partie du bien collectif. Pour être tout à fait clair, on doit reconnaître que dans certaines structures - une prison répressive ou une communauté anomique, une usine perturbée par un conflit, une faculté en proie à des rivalités - , ce «bien» peut avoir un caractère manifeste d'oppression ou de souffrance en même temps qu'il a l'avantage tout à fait évident d'apporter le soutien que représente la reconnaissance des autres. Les qualités particulières de ce «bien», que certains membres de la société recherchent, peuvent en effet provenir d'une expérience douloureuse vécue par la communauté tout entière. La compréhension profonde de tels phénomènes nous

36. Je m'inspire ici de la conception de la solidarité développée par Émile Durkheim dans La Division du travail social, première publication en français en 1893. Voir particulièrement le chapitre II sur la solidarité mécanique et le chapitre III sur la solidarité organique.

37. On se reportera à Talcott Parsons, «A Revised Analytical Approach to the Theory of Social Stratification», op. cit.

38. Voir les thèses de Georg Simmel sur le membership et la participation aux cercles sociaux, dans Kurt Wolff, dir., The Sociology of Georg Simmel, New York, Free Press, 1950. 
conduit, comme nous le verrons plus loin, au cœur de la théorie durkheimienne des pathologies sociales.

On ne peut réduire le fait d'accomplir ses obligations à la notion de biens, même si des acteurs envisagent en certaines occasions le soutien social comme une fin en soi, et en d'autres, comme un moyen de faire progresser leurs propres intérêts. Remplir des obligations, parce que cela relève essentiellement de relations collectives et que cela repose sur la réciprocité, revêt un sens profond. Ce sens dépasse celui que bien des individus donnent au fait de remplir des obligations, c'est-à-dire seulement comme un moyen de satisfaire ses besoins. Ce qu'il faut considérer comme l'entité fondamentale, ce n'est pas le désir d'un individu, mais c'est l'obligation à laquelle obéit le membre d'un groupe. Comme Durkheim l'a souligné, les obligations liées à la solidarité créent une réalité d'une nature différente de celle des satisfactions individuelles ${ }^{39}$. Le fait que les obligations soient en général intimement liées à la poursuite de buts personnels n'infirme en rien la validité de ce principe durkheimien. En réalité, on devrait considérer la recherche d'influence et, avec elle, la capacité à voir son opinion et sa personnalité reconnues par les autres, comme une activité sociale universelle, indépendante des efforts accomplis sur le plan économique pour obtenir les moyens de satisfaire besoins ou désirs.

Le fait de remplir des obligations sociales est un fardeau. Les gens peuvent bien dire qu'ils sont heureux de faire quelque chose pour un ami ou pour un collègue et que cela ne représente pas un effort réel. De telles attentions sont, au fond, plutôt l'expression d'un sentiment positif pour une autre personne. Mais accomplir un devoir exige, en général, un effort et du temps et peut empêcher de remplir d'autres devoirs. Généralement, quand il s'agit de remplir des obligations dans' des structures complexes, on se trouve devant des attentes contradictoires et des sources d'influence conflictuelles ${ }^{40}$. C'est le type de situations que les sociologues identifient comme des pressions contradictoires (cross-pressures) plus ou moins latentes. Dans ces cas-là, les décisions que l'on doit prendre pour remplir un devoir particulier vont entraver les efforts, réels ou potentiels, nécessaires à l'accomplissement d'autres obligations, obligations qui incombent à soimême ou à l'entourage.

L'accomplissement des devoirs face à des pressions contradictoires devrait être considéré comme typique du point de vue d'une théorie de la solidarité qui porte sur l'intégration sociale à grande échelle. Le fait d'accomplir des obligations selon la volonté d'un acteur qui exerce de l'influence a généralement comme contrepartie désagréable d'avoir à en décevoir d'autres qui détiennent eux aussi de l'influence et qui s'attendent à ce que l'on agisse différemment. On doit calculer le cours de sa conduite en fonction des devoirs que l'on a envers toute une série d'autres personnes, en envisageant l'avenir de nos relations avec elles. Agir sous l'influence d'une personne sous-entend généralement qu'on ait la force de résister à l'influence des autres et, dans une certaine mesure, qu'on mette en péril ses relations avec ces dernières. Agir par solidarité avec un acteur renforce vraisemblablement la relation avec lui, mais entame en général l'intensité des autres relations. À moins d'être capable de bien justifier de sa conduite, on peut perdre de l'influence sur les personnes que l'on a désappointées et en perdre peut-être aussi sur ceux et celles qui subissent l'influence de ces dernières. Utiliser son influence équivaut à «demander» à un ou plusieurs autres acteurs d'entrer dans une nouvelle relation de solidarité ou de remplir un devoir, dans la continuité d'une association ou d'une relation 1895

39. Émile Durkheim, Les règles de la méthode sociologique, première publication en français en

40. Voir Talcott Parsons, The Social System, New York, Free Press, 1951, particulièrement le chapitre XII, «Deviant Behavior and the Mechanisms of Social Control». Malheureusement, Parsons n'a jamais tenté de réanalyser de près ce point en le situant dans le contexte de la théorie de l'influence en tant que médium généralisé. 
de solidarité. Cela exige d'être en mesure de ranimer un lien de solidarité, une fidélité, de rappeler un devoir, une obligation. C'est une façon de se servir de son jugement pour mettre en branle des processus d'intégration et de solidarisation. Cela représente une «dépense» au sens où, en cherchant à coopérer avec les autres, on doit être prêt à leur livrer une part de l'influence que l'on détient en échange de leur soutien.

Pour plus de commodité, j'emploierai l'expression de prestation associative (associative performance) pour désigner le type de comportement qui s'exprime en réponse à l'exercice de l'influence. C'est parce qu'elle s'articule autour des liens de solidarité que la prestation associative diffère des diverses autres formes de conduite sociale, et non pas à cause de caractéristiques que l'on peut observer dans le comportement concret qu'elle entraîne. Tout comme un bien économique tire sa valeur non seulement de ses propriétés intrinsèques, mais de sa place dans un «univers» complexe d'utilités et de rapports à des buts culturellement légitimés, la prestation associative tire sa signification de tout un «univers» de liens de solidarité. On s'engage dans une prestation associative particulière pour accomplir les tâches qu'impose une relation de solidarité ou pour consolider les bases d'une nouvelle relation en respectant les responsabilités qu'elle fait naître. L'acteur doit déployer l'effort de volonté requis pour faire face à ses obligations. Le principal problème auquel on fait face lorsqu'on remplit une obligation est de redéfinir des priorités entre les devoirs à accomplir par rapport aux différents liens de solidarité. Quand on répond à l'influence d'une personne ou d'un groupe, on peut être contraint d'imposer un délai à la satisfaction d'attentes établies dans d'autres contextes, ou même de refuser catégoriquement de les assumer pour éviter de se retrouver dans des situations conflictuelles. En ce sens, redéfinir des priorités pose des difficultés propres aux acteurs qui sont engagés dans plusieurs rôles sociaux - c'est-à-dire presque tout le monde - et qui prévoient entretenir toute une série de liens de solidarité. La valeur que l'on accorde à la prestation associative tient le plus souvent à l'importance des priorités que l'on a modifiées en prévision de sa conduite à venir. On ne peut toutefois estimer la valeur de la prestation associative qu'en fonction d'un bénéfice immédiat ou qu'en fonction de l'efficacité de services concrets quoiqu'il puisse être important pour une association de compter sur des avantages pratiques. La valeur de la prestation réside essentiellement dans le fait qu'on ait consenti à agir pour prolonger dans l'avenir une relation de solidarité particulière et cela en dépit des «demandes» concurrentes liées à d'autres relations. En tant que catégorie générale, la prestation associative consiste à utiliser son jugement pour faire en sorte que la solidarité continue d'être une composante courante de la qualité des relations sociales.

Les «dépenses» d'influence, si elles sont rationalisées, reposent sur une série de décisions de fond que l'on peut, en gros, représenter par une courbe du style de celles qu'établissent les économistes. La courbe de l'influence a tendance à décliner vers la droite quand la quantité d'influence est reportée sur l'axe vertical et la quantité ou valeur de la prestation associative est reportée sur l'axe horizontal. Autrement dit, en «dépensant» une petite quantité d'influence, il y aura un accroissement de la demande de prestations associatives d'un type donné.

À l'inverse, la prestation associative est également soumise au jugement rationnel des acteurs qui l'offrent. Elle est représentée, tout au long de la courbe qui remonte vers la droite, par la quantité ou la valeur donnée qui augmente en fonction de la quantité d'influence susceptible d'être gagnée. Des courants, ou en d'autres termes des échanges particuliers (influence contre prestations associatives) se produisent à l'intersection des deux courbes projetées: celle des acteurs qui usent de leur influence et celle des acteurs y répondant. Pour analyser les «quantités» d'influence qui circulent, on doit prêter attention aux phénomènes qui sous-tendent ces deux courbes. Les coûts de la prestation, c'est-àdire les difficultés que rencontre celui qui la donne pour maintenir des relations de solidarité affectent la quantité d'influence «dépensée», autant que les réserves ou fonds d'influence dont clispose l'acteur qui la réclame. 
FIGURE 1

Courbes «normales» pour l'influence et la prestation associative

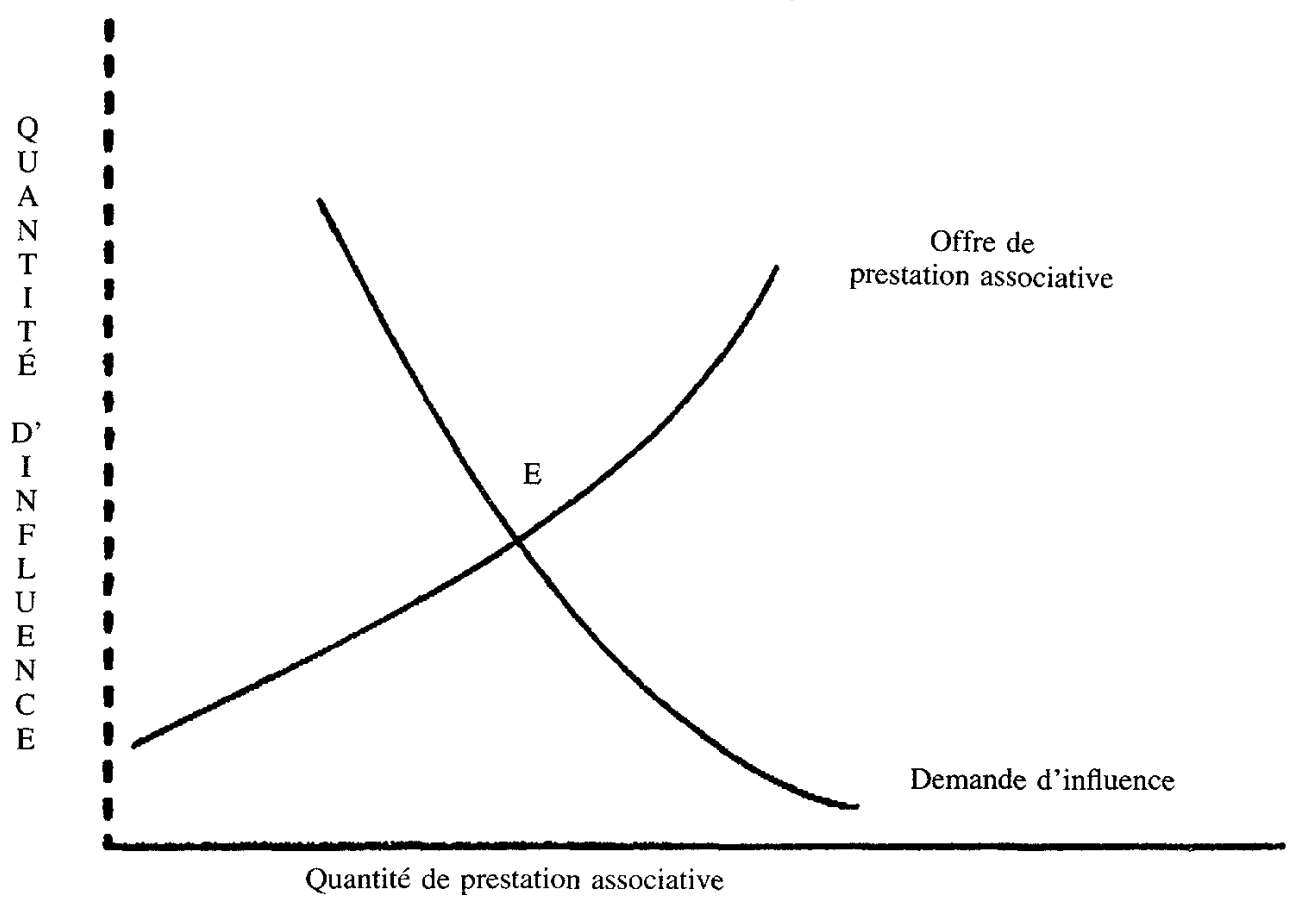

La figure $1^{41}$ montre l'intersection, au point $E$, d'une courbe ascendante d'influence avec une courbe déclinante de prestation associative. Le point $\mathrm{E}$ représente la condition d'équilibre hypothétique. Cet équilibre sera atteint dans l'ensemble pour autant que les membres du système de solidarité en question jouissent de la liberté d'association. Comme Parsons l'a lui-même souligné, la liberté d'association constitue, pour les relations d'intégration, l'équivalent du libre marché compris comme l'ensemble complexe d'institutions qui structurent l'économie ${ }^{42}$. Toutefois, la liberté d'association ne découle pas seulement de l'absence de contraintes normatives qui pèseraient sur les relations de solidarité. De la même manière que le libre marché s'appuie sur des institutions comme la propriété et le contrat, la liberté d'association exige une régulation effective des liens d'intégration. Des institutions comme les principes de la liberté des citoyens doivent garantir aux individus qu'ils peuvent s'exprimer librement tout en gardant l'espoir que les autres tiendront les engagements qu'ils ont pris de remplir leurs obligations.

En fait, dans une société complexe, il existe une foule de lieux propices à la formation de liens associatifs. Les communautés locales, les réseaux de parenté, les associations professionnelles, les groupes d'intérêts industriels, les regroupements de citoyens, les associations de charité et autres possèdent des structures indépendantes à l'intérieur desquelles s'échangent de l'influence et des manifestations de solidarité. Ces structures existent aussi dans les organisations où des liens peuvent se développer entre de tels groupes. Ces sphères de solidarité, différenciées, segmentées, et donc distinctes les unes des autres,

41. Pour la conception des figures présentées dans cet article, j'ai eu recours à mon fils, Larry Lidz, qui le premier s'est aventuré à les dessiner sur écran cathodique.

42. Voir T. Parsons, «Full Citizenship for the Negro American?», op. cit. Ce thème est développé de façon beaucoup plus complète dans The American Societal Community, op. cit. 
sont toutes formées dans des conditions sociales particulières ${ }^{43}$. Certaines de ces conditions seulement permettent une approximation de l'idéal-type de la liberté d'association. De nombreuses structures renferment des forces sociales qui détournent les processus associatifs de l'idéal de liberté. Les citoyens d'un quartier pauvre, qui vivent l'oppression de la misère ou qui redoutent la délinquance de la rue, ne peuvent pas s'associer en toute liberté. Les organisations militaires et les services de renseignements restreignent la liberté d'association de leur personnel. En général, la vie de famille suppose que la liberté des conjoints est limitée quand il s'agit de nouer des relations personnelles, et en particulier des relations intimes avec d'autres personnes; est également limitée la liberté des enfants à agir sans tenir compte du jugement «fiduciaire» de leurs parents. Dans le processus politique, les groupes de pression les plus puissants sont en mesure d'établir des oligopoles d'influence qui empêchent les groupes concurrents de se faire entendre dans les centres de décision ou de délibération politique.

La Démocratie en Amérique de Tocqueville est un classique de l'étude du rôle décisif joué par la liberté d'association dans le développement de la civilisation moderne. Tocqueville a établi la comparaison entre la société démocratique et la société aristocratique sous l'optique de la liberté d'association ${ }^{44}$. Dans la société aristocratique, tous les individus sont rattachés par leur naissance à des blocs sociaux qui définissent leurs devoirs à l'endroit de la société. L'aristocrate doit assumer le leadership de son groupe social et représenter les intérêts de la communauté à laquelle il appartient dans la société en général. Si l'on est du commun, on ne peut exercer d'influence que sur un plan local et suivant les voies établies par la tradition. Pour intervenir auprès de corps politiques plus importants, on doit s'adresser avec déférence à l'aristocrate dont on dépend et l'amener à agir selon ce que l'on souhaite. La société démocratique, par contre, place ses membres dans la position de citoyens autonomes et égaux. Tocqueville a insisté sur le fait qu'en l'absence de liens hérités auxquels se fier, les citoyens d'une démocratie sont fondamentalement faibles et seuls. Ils n'ont aucun canal d'influence durable leur permettant d'obtenir un soutien social ou d'exercer leur volonté. Afin de compenser cette faiblesse, ils sont amenés à s'investir avec énergie et créativité dans plusieurs espaces associatifs. Ils ont sans cesse à créer de nouvelles relations de solidarité grâce auxquelles ils peuvent affirmer et défendre leurs intérêts sociaux. Les associations se multiplient et l'influence circule beaucoup plus rapidement et plus largement. Bien que Tocqueville les ait construits de façon assez sommaire, ces modèles des sociétés aristocratique et démocratique dépeignent sans doute correctement la différence essentielle entre les «marchés» restreints de l'influence dans l'Europe pré-moderne et les marchés extrêmement actifs des sociétés égalitaires contemporaines. La transition de la société aristocratique à la société démocratique a opéré un changement fondamental dans le système de solidarité, changement qui a largement permis de développer le pluralisme des relations liées au rôle social et une énergie consacrée aux liens d'association.

Durkheim a formulé une idée analogue en des termes qui, selon notre interprétation, mettent l'accent sur les équilibres d'ensemble de l'influence et de la prestation associative. Dans le livre II de La Division du travail social, Durkheim a soutenu que le passage à une plus grande confiance dans les relations organiques et à la division du travail à l'époque moderne a été favorisé, dans la période précédente, par une densité morale ou

43. Les écrits de Simmels sur la formation des cercles sociaux dans les sociétés modernes constituent la principale référence classique en sociologie pour examiner l'ensemble des articulations entre les sphères indépendantes d'influence. Voir The Sociology of Georg Simmel, op. cit. et «Group Expansion and the Development of Individuality» dans Donald N. Levin, dir., Georg Simmel on Individuality and Social Forms, Chicago, University of Chicago Press, 1971.

44. Alexis de Tocqueville, De la démocratie en Amerique, vol. 1, première édition en français en 1835. Voir particulièrement le chapitre III pour la première formulation conceptuelle des types de société démocratique et aristocratique. 
densité dynamique croissante ${ }^{45}$. Par densité dynamique, Durkheim entendait l'engagement direct de groupes et d'individus dans des champs unifiés d'interaction où, selon un cadre normatif commun, ils seront en mesure de développer des liens de solidarité. La densité dynamique augmente quand, par le développement de conceptions plus abstraites et plus répandues de l'identité morale, les frontières institutionnelles qui divisent une société en de nombreuses communautés plus étroitement segmentées s'effondrent pour être alors remplacées par des cadres culturels établissant des groupes de solidarité plus larges, plus englobants.

Durkheim a observé ce processus à différentes périodes de l'histoire qu'il a classées à partir de la consolidation de la poleis dans la Grèce antique jusqu'à la formation de nations suprarégionales à l'époque moderne, en passant par l'intégration de nombreux "peuples» à l'Empire romain, à la fin de l'Antiquité. Il a cherché à montrer que, dans chaque cas, la vie sociale a été réorganisée moins à cause d'une extension du volume de population qu'à la suite d'un accroissement de la densité dynamique. Certaines sociétés traditionnelles, comme la Chine ou l'Inde pré-modernes, ont atteint des proportions considérables tout en demeurant attachées à un modèle de solidarités dispersées, segmentaires, centrées sur le village. D'autres sociétés, petites par leur volume mais denses par leur structure - par exemple les Pays-Bas ou la Belgique - ont néanmoins développé des formes modernes de solidarité fondées sur une plus grande liberté d'association. Les changements décisifs à l'époque moderne ont, par conséquent, été associés de façon spécifique à la croissance de la densité dynamique. Seule cette dernière conduit les membres d'une société à établir des contacts plus directs et à développer une dépendance mutuelle. Sur le plan économique, cela entraîne une intensification de la concurrence et la nécessité, pour les producteurs, de se démarquer les uns des autres. La division du travail s'en trouve étendue. Par conséquent, du point de vue de l'intégration, les individus et les groupes se trouvent liés dans un ensemble commun de «marchés» où s'échangent influence et prestation associative. Les membres de la société doivent alors développer un ensemble plus étoffé et plus souple de liens associatifs pour se sentir effectivement soutenus et reliés de façon adéquate les uns aux autres, dans leur vie sociale quotidienne.

Durkheim a décrit cet enrichissement de la division du travail et de la vie associative comme étant caractéristique de l'intensité croissante de la civilisation ${ }^{46}$. À la fin du Livre II et dans le Livre III de La Division du travail social, il a cherché à démontrer que cette intensité croissante répond à une augmentation de la densité, laquelle se développe de la même manière en quelque sorte spontanée qu'Adam Smith a observée lorsque la division du travail s'ajuste à un accroissement préalable du marché. Comme on peut le voir dans la figure 2, l'accroissement de la densité entraîne un déplacement vers l'extérieur de la

45. L'interprétation du concept durkheimien de densité morale ou dynamique a créé beaucoup de confusion. Notre interprétation est fondée sur le rejet de l'idée de Parsons selon laquelle l'explication de Durkheim de l'accroissement de la division du travail et de la solidarité organique faisait ressortir, en dernière analyse, la pression exercée par la population et était donc d'ordre «biologique» (The Structure of Social Action, p. 325); comme nous refusons également le point de vue développé par Mark Traugott dans son «Introduction» à Émile Durkheim on Morality and Society, Chicago, University of Chicago Press, 1978, et élaboré par Jeffrey C. Alexander, dans The Antinomies of Classical Thought: Marx and Durkheim, qui considère que Durkheim a proposé une explication «matérielle». Selon ma compréhension, qui ne peut être totalement justifiée ici, Durkheim a souligné que le facteur causal déterminant est la densité «morale» qui représente les liens de solidarité maintenant les membres de la société à l'intérieur d'un réseau unifié de relations. Cette explication est tout à fait en accord avec l'accent mis sur la culture morale et sur les institutions sociales dans le concept le plus essentiel des premiers travaux de Durkheim, la conscience collective. Durkheim souligne la densité «matérielle» parce qu'elle est plus facilement observable et mesurable que la densité «morale» et qu'elle paraît fournir un indice approximatif des états du facteur dynamique sous-jacent. En accord avec ce schéma d'analyse, Durkheim emploie la densité «dynamique» comme un autre terme pour exprimer la densité morale, non la densité matérielle.

46. Voir en particulier Émile Durkheim, La Division du travail social, Livre II, chapitres IV et V. 
FIGURE 2

Écarts dans les courbes de l'influence et de la prestation associative représentant un accroissement (développement) du système des relations de solidarité

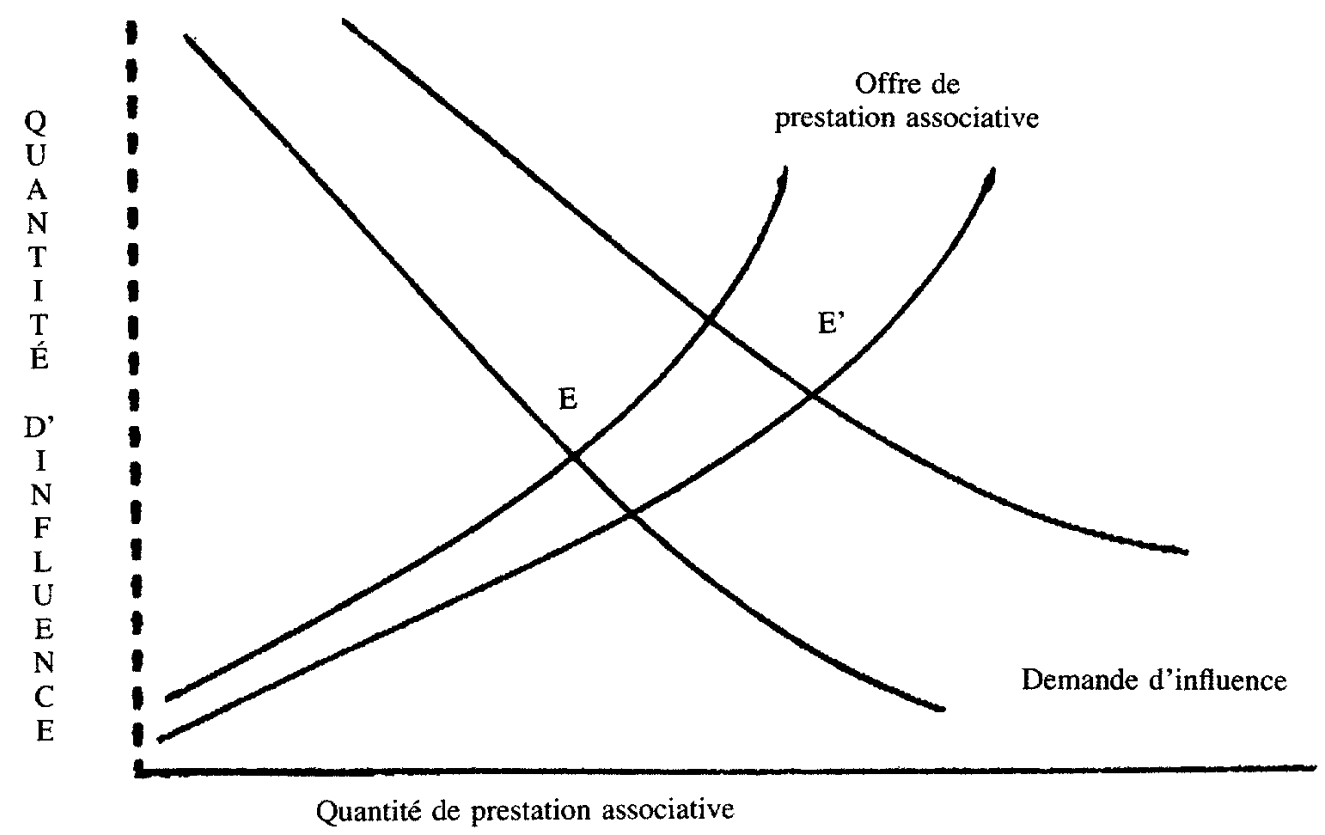

courbe de l'influence, alors que l'accroissement de l'intensité provoque le même type d'écart pour la courbe de la prestation associative. Ensemble, les écarts de ces deux courbes de l'influence et de la prestation associative déplacent le point d'équilibre vers E. Ce nouvel équilibre est atteint à un point qui représente un degré plus élevé d'opérations d'intégration ou de solidarisation et indique la croissance du système d'intégration.

\section{INFLUENCE ET INTÉGRATION SOCIALE}

On peut clarifier le concept de l'influence considérée comme médium généralisé si l'on fait quelques brèves comparaisons avec la monnaie. On verra que, sous certains aspects, l'influence est assimilable à la monnaie alors que sous certains autres, elle en diffère en raison de son rôle spécifique dans l'intégration.

Comme les échanges entre monnaie et biens ou services, les échanges entre influence et prestations associatives ont un caractère essentiellement contractuel. La rationalité implicite des échanges entre influence et prestation associative, ainsi que nous l'avons exposé plus haut, suppose cette qualité contractuelle et dépend d'elle. Un acteur que quelqu'un d'autre cherche à influencer conserve le droit d'accepter ou de rejeter l'offre que celui-ci lui fait. La quantité d'influence qui est offerte peut être jugée insuffisante ou encore il se peut que la prestation associative attendue en retour engage une conduite que l'acteur ne veut pas ou ne peut pas assumer. Tout comme on peut refuser de vendre un objet ayant une valeur marchande, on peut s'abstenir de répondre à l'exigence de solidarité réclamée par une personne influente. Cette nature contractuelle ou volontaire de la relation d'influence diffère nettement de celle des relations fondées sur le pouvoir ${ }^{47}$.

47. Je pose en ces termes les différences entre la monnaie et l'influence, d'une part, le pouvoir et les engagements d'autre part, que Parsons, lui, dans «On the Concept of Political Power» et dans «On the Concept of Influence», op. cit., a posé en termes de sanctions positives ou négatives sur lesquelles repose la généralisation de chacun des médias symboliques. 
Quand on recourt au pouvoir pour donner un ordre, on impose des obligations aux gens sans tenir compte de leur avis. Sans doute conservent-ils le choix entre obéir et être pénalisés pour ne pas l'avoir fait et, dans ce sens, ils agissent de leur plein gré. Mais les obligations finissent en général par les contraindre, de façon formelle, en dehors de leur volonté. Ils n'ont pas vraiment le choix de ne pas accepter les obligations, à moins de désobéir ou de quitter la structure de pouvoir en question.

Comme nous l'avons déjà constaté à quelques reprises, l'influence s'exerce en général dans un contexte chargé d'autres influences qui sont réellement, ou potentiellement, concurrentes. L'influence est un médium qui, comme la monnaie, mais peut-être à la différence du pouvoir, s'adapte avec une certaine flexibilité aux situations de concurrence. La nature compétitive de l'influence en tant que médium souligne l'importance du groupe de référence qui structure les situations sociales. Qu'ils offrent ou qu'ils acceptent de l'influence, les acteurs finissent en général par savoir comment s'assurer du soutien des relations les plus avantageuses pour eux, parmi la multitude de possibilités offertes par les structures de leurs groupes de référence respectifs. Les acteurs sont donc sensibilisés, par les structures latentes ou sous-jacentes de leur groupe de référence, au fait que chaque contrat d'influence et de prestation leur donne l'occasion d'engager d'autres relations, prévisibles ou inattendues. Plus son groupe de référence possède une forte solidarité déjà établie, plus l'acteur sera préoccupé du soutien social qu'il risque de perdre en favorisant un lien concurrent ou conflictuel avec un autre groupe que le sien. On ne peut donc pas envisager la formation de relations de solidarité spécifiques sans faire intervenir des notions comme celle du «coût de risques» (opportunity cost).

Sur un point clé cependant, la formation des relations de solidarité diffère très nettement de la forme quid pro quo des contrats économiques. Cette différence tient à l'affaiblissement du potentiel compétitif et conflictuel du mécanisme de l'influence et à la stabilisation des recoupements de liens d'origines diverses. Dans le contexte économique, l'acteur qui vend les droits d'une propriété donnée peut stipuler dans le contrat qu'il ne les cède qu'à une seule autre partie. Il serait alors frauduleux de tenter de vendre les mêmes droits, pour les mêmes biens, à plus d'une partie. Si plusieurs acteurs doivent gérer les mêmes biens, on doit recourir, pour régler la question des droits, à des formes juridiques complexes, comme les condominiums ou les copropriétés. Par contre, quand des relations de solidarité sont en jeu, les «contrats» entre les parties ne sont pas forcément exclusifs. L'influence n'exige pas, à la différence de la monnaie, que les acteurs choisissent parmi les offres de solidarité auxquelles ils vont répondre. Une prestation adaptée peut, en même temps, répondre positivement à une pluralité d'influences. Tout politicien d'une grande ville qui a l'idée d'étendre les services de police dans le but d'assurer une plus grande sécurité aux petits propriétaires de la classe moyenne, tout en offrant de nouveaux emplois aux minorités pauvres, connaît bien ce principe formulé à partir du simple bon sens. La solidarité peut être consolidée si l'on trouve une mesure qui réponde aux influences concurrentes et évite le conflit ou une possibilité de conflit.

Quand des collectivités ou des communautés complexes doivent élaborer une politique, on fait souvent des propositions pour tenter de concilier les intérêts des différents groupes qui ont exercé leur influence lors des concertations préalables. La possibilité que les prestations associatives créent de nouveaux liens de solidarité en faisant la promotion d'intérêts communs entre des groupes qui étaient jusqu'alors en concurrence, est la marque de leur fonction spécialisée d'intégration dans la vie sociale. Les sociologues ont peutêtre sous-estimé jusqu'à quel point le fait d'agir sous des pressions contradictoires est un trait positif de la vie associative, d'autant que cette capacité constitue un moyen important de limiter la compétition sociale. Agir sous des pressions contradictoires, bien que cela sous-entende des attentes également contradictoires et une certaine tension sociale, n'est généralement pas aussi paralysant pour la créativité et la détermination que certains sociologues l'ont laissé entendre. Le leader politique qui décide d'un moyen d'action en 
réponse à différentes sources d'influence a des chances d'acquérir une influence plus grande. Dans des situations où les politiques jouent un rôle important et où les groupes sociaux sont vraiment diversifiés, l'influence d'un leader peut devenir considérable au point de lui procurer - comme à un Franklin Roosevelt après les succès politiques du New Deal, par exemple - une place à peu près inattaquable dans le cœur de l'ensemble des citoyens. De grands défis de la vie publique peuvent être relevés par une conception créative des prestations associatives, dans la mesure où l'on a la possibilité de conclure des «contrats» politiques avec un large éventail de groupes.

Depuis Tocqueville, évoquer le fait qu'aux États-Unis les leaders répugnent à s'aliéner toute source importante d'influence au sein de la population est devenu un cliché sur la vie politique américaine. Toutefois, des rejets d'influence se produisent vraiment dans la vie politique et y jouent parfois un grand rôle. Il arrive que certains groupes soient écartés du débat public quand leurs besoins et leur influence paraissent difficiles à satisfaire sans devoir s'aliéner d'autres groupes qui, par leur influence, ont déjà exercé un certain contrôle sur les politiques publiques. Une telle privation du droit de participation s'observe en particulier quand le cadre normatif dans lequel sont établis les modes d'usage de l'influence de la classe moyenne risque d'être remis en cause par une nouvelle formulation des besoins et des influences. Les autorités américaines ont trouvé plus simples, ces dernières années, d'ignorer les sans-abris, les déficients mentaux, les familles d'assistés sociaux et les toxicomanes des quartiers pauvres, plutôt que d'abandonner les points de vue moralistes de la classe moyenne qui sous-tendent la politique sociale et qui répondent aux besoins de cette classe.

L'influence semble également se distinguer de la monnaie par le fait qu'elle circule dans des formes différenciées qui ne peuvent pas être facilement assimilées les unes aux autres. De par la nature de l'argent et des procédures comptables dans une économie moderne, l'argent liquide, les chèques, les dépôts bancaires, et même les lignes de crédit, sont en fait équivalents. S'il est commode pour un acteur $A$ de donner à un acteur $B$ un chèque de $100 \$$, et pour l'acteur $B$ de lui rendre cette somme en liquide, ils auront échangé des montants équivalents à condition qu'aucun intérêt ne coure à cause d'un retard dans le retour du paiement. De plus, cette équivalence existe quelles que soient les différences entre les rôles sociaux de $A$ et de $B$. Deux mêmes montants d'argent sont formellement équivalents, indépendamment de qui les possède ou les dépense, qu'il s'agisse d'une entreprise commerciale, d'une agence gouvernementale, d'une fondation à but non lucratif ou d'un ménage. Tous les avoirs en argent sont interchangeables d'un point de vue formel, quel que soit le secteur d'activités dans lequel ils ont été gagnés ou dépensés. Toutefois, il y a des différences dans le cas de l'influence, précisément quant aux rôles distincts joués par $A$ et $B^{48}$. Si $A$ est médecin et $B$ politicien, l'un et l'autre vont avoir recours, dans leurs rôles professionnels, à des formes d'influence différentes et non interchangeables. Ce n'est pas parce que $B$ est placé sous l'influence de $A$ qu'il est pour autant habilité à conseiller des patients. Si $A$ subit l'influence de $B$, il ne sera pas, de ce fait, capable d'exercer son ascendant dans les affaires publiques. Il est des usages particuliers, cependant, pour lesquels on peut remettre en circulation de l'influence ayant déjà circulé une fois. Le leader politique pourra peut-être réutiliser l'influence du médecin en invoquant son nom, ses titres, son statut social, son prestige, etc., si une

48. Parsons a anticipé certains aspects de la discussion qui suit. Dans son premier article sur l'influence comme médium généralisé, il a souligné la différenciation de l'influence en des formes très diverses. Toutefois il a elu tendance à identifier ces formes aux sous-systèmes de la communauté sociétale. Voir Politics and Social Structure, op. cit., p. 419 et sq. Plus tard, il a analysé les formes spécifiques de l'influence comme étant liées au fonctionnement d'ensembles complexes d'institutions données telles les universités. Voir T. Parsons et G. M. Platt, The American University, op. cit. Dans ces discussions, la question de savoir comment la spécialisation entre les formes d'influence devrait être reliée d'un point de vue théorique à l'idée de base de la géréralisation du médium n'a pas été éclaircie. 
question touchant la politique de la santé est en jeu. Il peut également invoquer l'influence du médecin si, dans sa vie privée de citoyen, il désire que sa famille et ses amis l'aident à suivre un régime médical. Parallèlement, le médecin peut faire circuler à nouveau l'influence du politicien en sollicitant la coopération de certains de ses collègues sur une question politique relative aux conditions de la pratique médicale.

Dans des cas de ce type, l'influence remise en circulation doit faire pression sur l'organisation sociale engagée dans la zone d'influence par le réutilisateur de celle-ci. Le second utilisateur peut être désigné sous le terme d'utilisateur par dérivation (derivative user). Dans les situations nouvelles, les critères qui réglementent les usages de l'influence sont souvent différents. Par exemple, le médecin influent qui souhaite agir sur les politiques de santé publique et le politicien qui voudrait citer les points de vue de ce médecin vont devoir affronter les règles du débat politique et non celles des instances qui régissent les soins médicaux. De plus, certains aspects pratiques d'une nouvelle structure peuvent différer des critères établis par la norme. Les structures dans lesquelles les médecins exercent en général leur influence sont caractérisées par un soutien mutuel entre collègues et des hiérarchies décisionnelles assez nettement définies. Les politiciens agissent le plus souvent dans des situations plus compétitives et moins fortement contraignantes. Si on les compare avec des médecins qui usent de leur influence dans un hôpital, les politiciens, eux, doivent prévoir jusqu'où les porte-parole de l'opposition peuvent se donner libre cours dans leurs discours et leurs défis. Opérer la transition d'un style d'influence à un autre et d'une structure d'influence à une autre se révèle souvent difficile, comme bien des autorités scientifiques du monde médical en ont fait l'expérience lors de leurs interventions dans le domaine législatif.

Les conditions de la remise en circulation de l'influence semblent être soumises à des contraintes supplémentaires liées au statut professionnel spécialisé des acteurs en cause. Si un politicien subit une influence dans le domaine de la médecine, il ne la réutilisera correctement que lorsqu'un autre politicien (premier ensemble de contraintes liées au statut) invoquera fort à propos une influence portant sur des questions médicales (deuxième ensemble de contraintes liées au statut). Cela implique que l'influence ne peut pas vraiment être acceptée ou reçue comme un chèque peut être déposé dans un compte bancaire, car même si on les «dépose», les formes différentes que revêt l'influence ne peuvent pour autant être confondues. De ce fait, les acteurs qui essaient d'exercer de l'influence en parlant au nom de groupes de solidarité, subissent de nombreuses pressions d'intégration. Ils doivent tenir en réserve des formes d'influence diverses, facilement disponibles pour être remises en circulation en cas de besoin. Pour ce faire, ils doivent entretenir des liens de solidarité avec un éventail de groupes qui ont de l'influence dans les différents milieux sociaux qui pour eux ont de l'importance. En assurant leur propre influence de cette façon, ils tendent à protéger, par effet de réciprocité, l'efficacité d'autres personnes qui sont influentes dans les mêmes réseaux de solidarité.

On ne devrait pas considérer que la spécialisation dans l'une des formes d'influence soit un obstacle à ce que celle-ci se généralise. L'influence semble se généraliser en fonction de la capacité d'intégration des communautés dans laquelle elle est reconnue. Qu'une forme d'influence soit spécialisée ne diminue pas le fait qu'elle se généralise; celle d'un médecin, par exemple, atteint un niveau élevé de généralisation, si ce médecin est professionnellement réputé et respecté par toute la communauté médicale du pays, ou par la société tout entière quand des questions de santé publique sont en jeu. Ainsi, le regretté $\mathrm{D}^{r}$ Paul Dudley White, qui a joué un rôle clé en établissant que l'exercice physique pouvait permettre à l'organisme de récupérer après des attaques cardiaques et pouvait même les prévenir, jouissait d'une influence qui dépassait sa spécialité dans le domaine de la cardiologie. De plus, un aspect crucial de la généralisation de l'influence tient à la capacité d'acceptation réciproque de ses diverses formes dans des groupes de référence différenciés et segmentés. L'influence professionnelle et l'influence politique ne peuvent 
être réduites l'une à l'autre, mais si l'une et l'autre circulent à travers une grande diversité de liens de solidarité, les deux sont fortement généralisées.

\section{5. ÉQUILIBRE NORMAL ET PATHOLOGIES SOCIALES}

En travaillant sur la notion d'un équilibre spontané de la solidarité entre la densité morale et l'intensité des liens sociaux dans une société, Durkheim a reconnu l'importance de nombreux facteurs qui peuvent le perturber. Il a voulu montrer à quel point il est difficile de préserver un équilibre vraiment spontané, dans lequel les membres de la société agissent librement mais de façon responsable, de manière autonome mais en toute loyauté à l'égard des autres. L'équilibre spontané peut être menacé par des circonstances diverses, particulièrement en des temps où les changements sociaux sont rapides et où l'on assiste à l'éclatement des institutions les plus importantes ${ }^{49}$. En poursuivant l'analyse de ces conditions et en révisant ses premières thèses, Durkheim a, dans Le Suicide, classé en quatre catégories les facteurs qui perturbent les équilibres de solidarité ${ }^{50}$. Ces quatre catégories constituent les types généraux de «pathologies sociales»: l'égoïsme et l'altruisme, l'anomie et le fatalisme.

De nombreux sociologues, et notamment Parsons lui-même ${ }^{51}$, ont interprété le schèrne des pathologies sociales comme étant essentiellement fondé sur les jugements de valeur personnels de Durkheim. Ils ont suggéré que les conditions sociales que Durkheim qualifie de pathologiques ne sont pas en soi meilleures ou pires que d'autres, mais qu'elles sont simplement conçues pour apparaitre ainsi dans la vision idéologique de Durkheim. Les gens qui participent de cultures différentes de celle à laquelle Durkheim s'est identifié peuvent donc valoriser plus l'une ou l'autre des conditions pathologiques que l'état d'équilibre normal, spontané. Les Américains peuvent peut-être effectivement préférer un certain degré d'égoïsme et peut-être même d'anomie. Les Soviétiques, par contre, peuvent privilégier un certain degré d'altruisme et de fatalisme. Dans ce sens, il est impossible de justifier, en termes sociologiques, que les conditions de l'équilibre spontané établies par Durkheim soient assimilées à la «normalité».

À l'encontre de cette interprétation établie, nous souhaitons proposer une explication différente de l'analyse durkheimienne. Durkheim a établi une analogie très précise entre la solidarité normale, résultat de l'équilibre spontané, et la notion économique de l'équilibre que ll'on atteint dans un marché libre et concurrentiel. Dans le modèle économique, chaque force qui perturbe les processus d'offre, de demande ou d'intégration du marché, va produire un équilibre qui sera moins efficace pour satisfaire les besoins des membres de la société. Durkheim a conçu ses catégories de pathologies sociales comme une simple classification des forces qui entraînent l'équilibre des relations de solidarité loin de son point optimum théorique. Une pathologie sociale est donc l'analogue d'une force économique qui empêcherait un marché de franchir son point de rendement optimal. Une pathologie sociale altère les processus qui permettent d'atteindre l'équilibre dans un champ particulier de relations sociales et diminue la solidarité effective qui va naître de cet équilibre.

D'un point de vue théorique général, l'idée de pathologie est à la fois précise et n'implique pas de jugement négatif, bien que cela soit difficile à saisir dans des études empiriques spécifiques. Durkheim a lui-même reconnu que les conditions sociales qu'il qualifie de pathologiques sont directement liées à des valeurs sociales positives. En cherchant à montrer que l'éthique sociale qui privilégie le progrès est une source importante

49. C'est la problématique générale du Livre III de La Division du travail social, op. cit.

50. Émile Durkheim, Le Suicide, première édition en français en 1897. Le concept de suicide fataliste, qui fait pourtant partie du schéma d'analyse de Durkheim, n'est mentionné qu'à l'occasion d'une note, p. 276.

51. On se reportera à The Structure of Social Action, op. cit., p. 371 et sq. Parsons n'a jamais voulu revenir sur cette critique sévère du concept durkheimien des pathologies. 
d'anomie, Durkheim n'avait pas véritablement l'intention d'en faire la critique. Il voulait plutôt souligner les complexités de la vie sociale en mettant en évidence le coût humain des valeurs qui constituaient le cœur du système de croyance qui était le sien ${ }^{52}$.

Nous devons aussi reconnaître que l'idée durkheimienne d'un équilibre normal ne suppose pas que chaque communauté, chaque sphère de relations sociales et chaque société doivent instituer le même modèle de solidarité. Deux considérations sont à prendre en compte concernant ce problème. Premièrement, pour Durkheim, l'équilibre normal est un équilibre spontané, et donc en constant changement, entre un état de densité et un état d'intensité. Des facteurs profondément enracinés dans la culture morale d'une société agissent sur son état de densité. Les conditions d'intensité sont déterminées par des modèles établis de relations de solidarité. En conséquence, le point d'équilibre normal résultera toujours d'un ajustement momentané aux caractéristiques, très institutionnalisées, mais cependant en évolution, d'une société spécifique. Deuxièmement, les différents domaines institutionnels d'une société donnée auront des conditions particulières de densité et d'intensité et, par conséquent, ressentiront le besoin d'atteindre un équilibre particulier de solidarité. Les institutions militaires et universitaires, les familles et les entreprises, les communautés locales et les secteurs industriels de pointe, les régions d'un État, les classes sociales et les groupes de statut vont tous adopter des modèles de solidarité quelque peu différents. C'est l'imbrication complexe de nombreuses formes différentes de solidarité qui constituera l'équilibre sociétal global. Dans de nombreuses structures, les déviations par rapport au point d'équilibre normal seront fortement valorisées. Ces déviations sont souvent enracinées dans les nécessités «fonctionnelles» d'institutions de type spécifique - le besoin de discipline chez les militaires, le sens du dévouement du personnel infirmier, la nécessité d'opérations boursières pour les individualistes à la recherche de profit.

La figure 3 utilise les courbes de l'influence et de la prestation associative pour interpréter avec plus de précision les quatre types durkheimiens de pathologie sociale. Deux de ces pathologies, l'égoïsme et l'altruisme, ont été analysées comme des conditions qui altèrent la configuration de la courbe de l'influence. L'anomie et le fatalisme ont été perçus comme des conditions qui déforment la courbe de la prestation associative. L'égoïsme et l'altruisme sont des pathologies qui affectent les conditions de la demande de solidarité, alors que l'anomie et le fatalisme agissent sur les conditions de la formation et de l'offre de solidarité.

L'égoïsme est une condition dans laquelle les acteurs n'ont pas besoin, pour s'associer, d'être fortement identifiés les uns aux autres dans une entité collective, mais doivent pouvoir créer leurs liens mutuels sur une base qui privilégie leur intérêt personnel. Dans les structures égoïstes, les facteurs institutionnels provoquent une chute plus rapide et plus brutale de la courbe de l'influence au moment où elle commence à décliner vers la droite. Celui qui exerce l'influence fait appel à l'intérêt individuel, parfois éclairé, des acteurs. Cette influence n'aboutit pas à l'intégration solide d'unités sociales à l'intérieur d'une collectivité ou d'une communauté et la faiblesse de la solidarité qui en résulte est de nature pathologique pour les gens qui ont besoin de compter sur des associés fiables. Les acteurs égoïstes «dépenseront» de l'influence, qui pourra être utilisée par la suite à les inciter à accomplir leurs devoirs, seulement si la quantité de prestation associative obtenue en échange de cette influence est très grande. Dans la société américaine, on trouve souvent cet égoïsme dans le monde du petit commerce indépendant où la plupart des comportements sont inspirés par un solide sens de l'intérêt personnel.

Dans l'altruisme, par contre, il faut qu'une forte identification mutuelle soit posée comme condition essentielle de l'association. Ce qui inspire la conduite, c'est la volonté d'agir dans l'intérêt collectif. L'altruisme concerne les conditions dans lesquelles la courbe de l'influence est déviée vers le haut et décline lentement, de telle sorte que les liens

52. Voir Le Suicide, op. cit., particulièrement le Livre III, chapitre 3. 
communautaires doivent être puissants pour mettre en œuvre les relations de solidarité. On va dépenser plus d'influence pour obtenir des prestations. L'acteur qui répond à l'influence obtiendra, en retour de l'acquittement de ses devoirs, une capacité plus grande à faire éventuellement intervenir les liens réciproques de solidarité. Les usages altruistes de l'influence soulignent le besoin de participation au bien collectif ou communautaire, en impliquant un levier plus puissant pour faire jouer les obligations réciproques. Mais l'altruisme peut être vécu comme une pathologie par les gens qui, contraints en permanence à agir dans l'intérêt collectif, finissent par perdre leur capacité de jugement et d'action autonome. L'altruisme dans sa forme modérée ressemble à ce que Parsons a appelé, à la fin de sa carrière, le caractère fiduciaire des rôles parentaux dans la famille. Pour s'occuper de leurs enfants et permettre leur socialisation, les parents ont le devoir de subordonner leurs intérêts immédiats au bien-être de la famille et aux intérêts à long terme de la génération qui les suit.

L'anomie apparaît quand les contraintes sociales qui établissent les règles des liens d'obligations réciproques entre les acteurs sont affaiblies. On peut formaliser l'anomie comme la déviation vers le bas à droite de la courbe de la participation associative de façon telle qu'elle ne remonte que lentement de l'axe horizontal. C'est là le schéma qui se dessine quand la participation peut être obtenue facilement et à moindres frais en termes des obligations collectives que suppose l'influence, et quand les «coûts» de la demande de solidarité sont bas en raison de l'affaiblissement de conditions implicites de celle-ci. Bien que des associations puissent tout de même se former dans des conditions anomiques et que l'anomie, comme le reconnait Durkheim, encourage l'innovation dans la vie sociale, les acteurs risquent de subir comme une pathologie la fragilité et la non-calculabilité des contraintes sociales. Celui qui essaie d'influencer les autres peut avoir souvent des doutes sur le fait que ceux-ci reconnaîtront qu'ils ont des devoirs. Comme Durkheim l'a souligné, l'anomie est souvent présente dans les industries et dans les sciences qui évoluent de façon constante, où les critères de travail sont ouverts à des changements rapides, qui imposent sans cesse des réorientations.

Le fatalisme, c'est le renforcement considérable des contraintes sociales. Il caractérise les situations dans lesquelles la courbe de la participation s'élève brusquement pour devenir presque parallèle à l'axe vertical. Les liens et les obligations générés par la solidarité sont forts et les conséquences de toute prestation associative sont déterminantes, lourdes et entraînent des «coûts» élevés. On peut obtenir des prestations spécifiques seulement si l'on engage de grandes «dépenses" d'influence, produisant une grande capacité, chez celui qui répond à l'influence, à faire intervenir par la suite des liens réciproques de solidarité. Si le dilemme auquel doit faire face l'acteur dans les situations d'anomie consiste à se demander si sa conduite aura des conséquences déterminées ou déterminables dans ses relations de solidarité, celui que doit affronter l'acteur dans des situations fatalistes revient à évaluer s'il va oser agir, connaissant le poids des conséquences qui peuvent s'attacher à la poursuite de son action. On atteint une forme pathologique de fatalisme quand le fait d'avoir à exercer son jugement représente un fardeau si lourd que l'on ose à peine agir. Dans les sociétés contemporaines, les prisons à haute sécurité sont peutêtre les plus fatalistes des structures institutionnelles de la vie sociale.

La figure 3 montre quatre points d'équilibre aux intersections des courbes des déviations pathologiques de l'influence et de la prestation associative. Les courbes ont été tracées afin de souligner les différences hypothétiques entre les quatre modèles distincts de pathologie sociale.

\section{a) LA SOLIDARITÉ FATALISTE-ALTRUISTE}

La vie militaire et la vie monastique peuvent constituer des exemples de cette forme de solidarité. La quantité de solidarité y est limitée mais les liens qui la composent sont 
FIGURE 3

Courbes «pathologiques» de

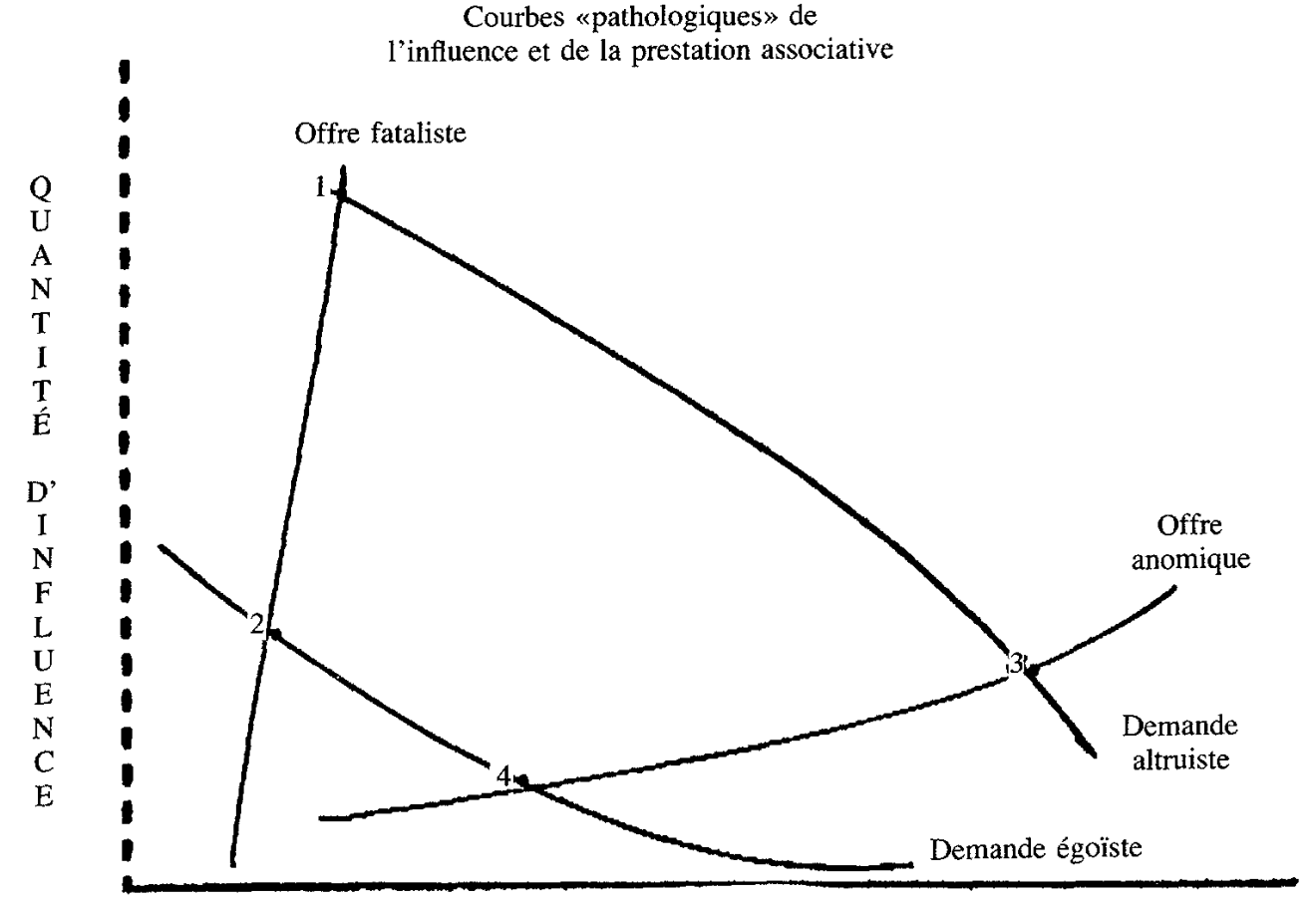

Quantité de prestation associative

solides et fiables, même s'ils exigent une grande discipline de la part de ceux qui en participent.

\section{b) LA SOLIDARITÉ FATALISTE-ÉGOÏSTE}

Celle-ci peut être représentée par le modèle que l'on retrouve couramment dans le milieu de la criminalité où les relations avec les autres sont soutenues par des contraintes strictes, extrêmement fortes, mais où l'on peut également donner libre cours aux intérêts personnels.

\section{c) LA SOLIDARITÉ ANOMIQUE-ALTRUISTE}

On retrouve vraisemblablement ce modèle dans la pratique du travail social dans le contexte du système du bien-être social américain. La définition même de leur profession repose sur l'altruisme, mais en s'acquittant de leurs devoirs, les travailleurs sociaux sont engagés dans des relations qui impliquent que de nombreux partenaires différents manipulent les règles et les contraintes sociales.

\section{d) LA SOLIDARITÉ ANOMIQUE-ÉGOÏSTE}

Aux États-Unis, les tentatives des groupes d'intérêts et des associations de bénévoles d'exercer une influence dans le domaine du politique relèvent de ce type de modèle de solidarité. La compétition y est ouverte et libre, et on y entend s'exprimer des intérêts très divers, mais les niveaux de responsabilité qu'on y engage sont peu élevés.

D'un point de vue sociologique, l'intérêt qu'il y a à identifier des modèles généraux de solidarité, c'est de pouvoir construire des hypothèses quant aux différences observables, selon des conditions variables, dans le caractère dynamique de la solidarité et dans ses 
qualités au niveau de l'expérience. La quantité de solidarité générée dans un système fataliste-altruiste, par exemple, sera bien moindre que dans un système anomique-égoïste. Cependant, pour des individus engagés dans des relations spécifiques, la solidarité paraîtra plus forte dans le système fataliste-altruiste. Les courbes de l'influence et de la prestation associative, avec leurs déviations pathologiques, sont susceptibles de guider l'élaboration et l'interprétation d'hypothèses.

\section{CONCLUSION: QUELQUES PISTES DE RECHERCHE}

L'objet de cet exercice théorique était d'introduire, de façon directe et en peu de mots, un appareil conceptuel qui puisse servir de théorie de base à la sociologie. Cependant, à la différence des autres théories de l'acteur rationnel, ce schéma est censé faire le lien entre la richesse conceptuelle de la théorie générale de l'action et la tradition de l'analyse institutionnelle en sociologie. Beaucoup de travail est encore nécessaire pour être certain de tirer tout le bénéfice de cette richesse conceptuelle. Il nous a paru utile de conclure en énumérant simplement les principaux points qui devraient faire l'objet de recherches futures:

1. On devra étudier les forces institutionnelles qui déterminent les courbes de l'influence et de la prestation associative des relations entre individus, dans des secteurs différents de la société.

2. Des recherches doivent être entreprises sur l'interaction entre les mécanismes de la solidarité et le système du marché en économie, la structure de pouvoir du politique et les mécanismes qui conduisent les acteurs à s'engager pour des valeurs (value-commitment) du système fiduciaire. Ce sont des sujets complexes qui impliquent de nombreux éléments structurels de base de la société, par exemple les structures juridiques, les systèmes de classes et autres. Une thêorie dynamique de la solidarité devrait permettre aux sociologues d'analyser ces questions avec plus d'efficacité qu'elles ne l'ont été jusqu'à présent.

3. Les modèles de pathologie sociale et les déviations qui y sont reliées, établies par les courbes de l'influence et de la prestation associative, devraient servir à faire des études comparatives des institutions d'intégration. Par exemple, la société américaine semble être caractérisée par le modèle égoïste-anomique de l'intégration, tandis que la société japonaise a un mode d'intégration plus altruiste et fataliste. La France est plus altruiste que les États-Unis et probablement plus anomique que l'Allemagne. Des études sur l'Irlande suggèrent que le modèle de ce pays est peut-être du type égoïste et fataliste ${ }^{53}$.

4. On devra analyser, en suivant les perspectives de $\mathrm{Baum}^{54}$, les relations de solidarité au niveau de l'action générale, autant qu'au niveau du système social. Cette analyse permettra d'examiner ensuite les aspects affectifs et sentimentaux de l'engagement dans des liens de solidarité plus directement que ce que nous avons suggéré dans cet article.

5. On devra étudier les écrits de Simmel, Toennis et d'autres penseurs identifiés à la tradition phénoménologique, et s'en inspirer pour analyser le fond et la forme des différentes sortes de relations de solidarité.

Victor Lidz

35 Aberdale Road Bala Synwyd

Pennsylvania 19004

U.S.A.

53. Voir Nancy Scheper-Hughes, Saints, Scholars and Schizophrenics, Berkeley, California University of California Press, 1979.

54. Baum, «The System of Solidarities», op. cit. 


\section{RÉSUMÉ}

Pendant les vingt dernières années de sa vie, Talcott Parsons a soutenu que l'objet d'étude essentiel de la sociologie devait être l'analyse de l'intégration sociale. S'inspirant pour une bonne part du modèle durkheimien de la solidarité, Parsons a conçu cette intégration sociale en termes dynamiques comme la capacité d'un groupe, d'une association ou d'une communauté à préserver sa cohésion lorsqu'ils se trouvent confrontés à des situations de désordre et de conflit. Les lacunes de la théorie de l'influence, théorie qu'il a placée au centre de son analyse de l'intégration sociale, expliquent en partie que Parsons ne soit pas parvenu à imposer ce programme de recherche à l'ensemble du milieu sociologique. Cet article s'efforce de reformuler la théorie de l'influence et de consolider ses fondements en envisageant les relations d'intégration comme étant de l'ordre de la rationalité. On propose un modèle comparable à celui de l'offre et de la demande. Enfin, on met en évidence les liens de ce modèle avec la théorie du groupe de référence et avec la théorie durkheimienne de la solidarité et des pathologies sociales.

\section{SUMMARY}

For more than twenty years before his death, Talcott Parsons maintained that the disciplinary focus of sociology should concern the analysis of social integration. Following in part the model of Durkheim's concept of solidarity, Parsons conceived social integration in dynamic terms as the capacity of a group, association, or community to maintain coherence when confronted by forces of disorder and conflict. Parsons' failure to project this research program to the profession at large was due in part to shortcomings in the theory of influence that he placed at the center of his analysis of social integration. The present essay attempts to reformulate the theory of influence, especially by strengthening its foundations in a conception of rational conduct in integrative relationships. A model comparable to supply and demand analysis is proposed. The linkages of this model to reference group theory and to Durkheim's theory of solidarity and social pathologies are emphasized.

\section{RESUMEN}

Por mās de veinte años antes de su muerte, Talcott Parsons mantuvo que el foco disciplinario de la sociología concierne al análisis de la integración social. Siguiendo en parte el modelo del concepto de solidaridad de Durkheim, Parsons concibió la integración social en términos dinámicos como la capacidad de un grupo, asociación o comunidad para mantener coherencia cuando se ve confrontada por fuerzas de desorden y conflicto. La falla de Parsons al proyectar este programa de investigación sobre la profesión en general se debió en parte al defecto en la teoría de la influencia que él colocó al centro de su análisis de la integración social. El presente ensayo intenta reformular la teoría de la influencia, especialmente fortaleciendo sus fundamentos en una concepción de conducta racional en relaciones de integración. Se propone un modelo comparable de análisis de oferta y demanda. Se pone énfasis en la unión de este modelo con el grupo teórico de referencia y con la teoría de Durkheim de solidaridad y patologías sociales. 